\title{
Entropy of a correlated system of nucleons
}

\author{
A. Rios, A. Polls, and A. Ramos \\ Departament d'Estructura i Constituents de la Matèria, Universitat de Barcelona, Avda. Diagonal 647, E-08028 Barcelona, Spain \\ H. Müther \\ Institut für Theoretische Physik, Universität Tübingen, D-72076 Tübingen, Germany \\ (Received 31 May 2006; published 28 November 2006)
}

\begin{abstract}
Realistic nucleon-nucleon interactions induce correlations to the nuclear many-body system, which lead to a fragmentation of the single-particle strength over a wide range of energies and momenta. We address the question of how this fragmentation affects the thermodynamical properties of nuclear matter. In particular, we show that the entropy can be computed with the help of a spectral function, which can be evaluated in terms of the self-energy obtained in the self-consistent Green's function approach. Results for the density and temperature dependences of the entropy per particle for symmetric nuclear matter are presented and compared to the results of lowest order finite-temperature Brueckner-Hartree-Fock calculations. The effects of correlations on the calculated entropy are small, if the appropriate quasiparticle approximation is used. The results demonstrate the thermodynamical consistency of the self-consistent $T$-matrix approximation for the evaluation of the Green's functions.
\end{abstract}

DOI: 10.1103/PhysRevC.74.054317

PACS number(s): 21.65.+f, 21.30.-x

\section{INTRODUCTION}

Entropy is a key quantity in the study of the thermodynamical (TD) properties of fermionic systems. In the context of correlated Fermi liquids, the study of entropy has been triggered by the experimental and theoretical studies of ${ }^{3} \mathrm{He}$ [1-6]. The specific heat of this system is experimentally known to have a nontrivial temperature dependence [7] of the type $T^{3} \ln T$. Such a nonanalytical behavior can be seen to arise within Fermi liquid theory from the coupling between quasiparticles and incoherent spin fluctuations (quasiholes in the triplet state), which give rise to nonanalytical energy dependences in the self-energy [8]. These nonanalyticities are in fact a very general feature of all normal Fermi liquids and their existence is not related to the particular details of ${ }^{3} \mathrm{He}$ systems.

Hot symmetric nuclear matter (SNM), on the other hand, is an infinite fermionic system composed of nucleons at very high densities. In this ideal system, only the strong interaction among nucleons is considered and any other interaction, such as the electromagnetic one between protons, is neglected. The temperatures usually considered for this system are of the order of tenths of $\mathrm{MeV}$ and are somewhat small if compared with the typical energy scales of nuclear matter (e.g., the free Fermi energy at saturation density is about $\epsilon_{F} \sim 40 \mathrm{MeV}$, and thus $T / \epsilon_{F} \sim 0.5$ for $T=20 \mathrm{MeV}$, the highest temperatures considered here). We expect that a finite-temperature approach to SNM can fairly describe the properties of the hot environments that could exist either inside the cores of supernovae at the latest stage of their evolution [9] or in the collisions of heavy nuclei at intermediate energies [10]. The TD properties of these systems and, in particular, the entropy are important quantities for understanding astrophysical and heavy-ion-physics phenomena. In core-collapse supernovae, for instance, the evolution and dynamics occur at a fixed entropy per baryon [11]. Moreover, the entropy production in multifragmentation events in heavy-ion collisions is considered to be a crucial quantity for determining the mass fragment distribution [12].

Hot SNM has been usually studied in a mean-field approximation with effective phenomenological nucleon-nucleon $(N N)$ forces, such as the Skyrme or Gogny interactions $[13,14]$, or within a relativistic mean-field approximation [15]. Other many-body approaches that have been used in the study of SNM include lattice models [16] or three-loop calculations within chiral perturbation theory at finite temperature [17]. However, when dealing with realistic $N N$ potentials, more sophisticated many-body techniques are needed. The strong short-range repulsion and the tensor components of these potentials modify substantially the many-body wave function, which is no longer well described in terms of a free Fermi gas Slater determinant. Particle-particle correlations, for instance, are crucial for a correct description of SNM properties from realistic $N N$ potentials. The Brueckner-Hartree-Fock (BHF) approach accounts for such correlations by means of a summation of an infinite series of suitable ladder diagrams [18]. In fact, the BHF approximation arises from a welldefined expansion for the energy of a fermionic system at zero temperature, the so-called Brueckner-Bethe-Goldstone expansion [19]. At finite temperature, a similar summation can be achieved in the so-called Bloch-de Dominicis (BdD) approach [20]. However, this approach is not devised to reproduce the energy of the system. Instead, it aims at computing the TD grand potential and, from it, all the TD properties of the system. In particular, the entropy includes the correlations embedded in the approach [21].

Traditionally, however, the BHF approach has been extended to finite temperatures in a more naive way: The energy of the system is computed from a simple generalization of the $T=0$ formalism to finite temperature and the entropy of the system is computed from a mean-field expression [22,23]. Finally, let us also note that relativistic BHF-type calculations at finite temperature have also been reported in the literature $[24,25]$. 
A consistent treatment of correlations in quantum manybody systems requires the inclusion of particle-particle and hole-hole scattering terms. The usual approach is the so-called ladder approximation, or $T$-matrix approximation, in which an expansion of the single-particle Green's function in terms of diagrams is achieved. Such an expansion goes beyond the BHF approach in the sense that the propagation of both particles and holes are treated in the same footing. Within this approach, the propagator of a correlated fermion is not simply described in terms of one quasiparticle pole. Instead, the strength of a momentum state $k$ is fragmented over a wide range of energies. The process of finding the Green's function for such a many-body system is unavoidably self-consistent, since the propagation of a nucleon is affected by the interactions with the surrounding nucleons, which in turn are also described in terms of the Green's function. Although one can easily write down the diagrammatic expansion that gives rise to this self-consistent Green's function (SCGF), it has taken a lot of time to obtain a numerical solution of the full problem. A major well-known issue is related to the so-called pairing instability that appears in the zero-temperature formalism when the propagation of holes is considered [26-28]. Nevertheless, calculations have been performed at the SCGF level at zero temperature within different approximations: with a quasiparticle self-consistent approach [29], with a discretized parametrization of the spectral function [30], or with a simplified separable $N N$ interaction [31]. In all these approaches the final output is the single-particle spectral function of a nucleon inside matter, which can also be obtained from other many-body approaches, such as correlated basis function theory [32].

In the case of SCGF, a finite-temperature treatment has been the keystone for obtaining a complete numerical solution from a realistic $N N$ potential [33-35]. Once the self-consistent propagator is obtained in this method, one can easily obtain information on the microscopic properties (such as the momentum distributions, self-energies, or spectral functions of the nucleon) as well as on the bulk properties of the system (the energy per particle via the Galitski-Migdal-Koltun (GMK) sum rule, for instance [36,37]). For a complete TD description of the system, however, one should compute the relevant TD potential of a statistical quantum mechanical system (i.e., the free energy). A suitable calculation of the entropy is thus required if this formalism is to be used in any practical description of hot SNM. Here, we will follow the Luttinger-Ward (LW) approach [6,38], in which the grand potential is computed from the full single-particle propagator. An analysis of the properties of SNM within this formalism has been recently published by Soma and Bożek [39]. In the following we shall show that one does not need to compute TD quantities within a full LW approach as is done in [39], provided that some approximations for the entropy are valid. Finally, let us notice that the LW formalism has been widely used in other many-body physics problems, ranging from relativistic plasmas [40] to resonances in heavy-ion collisions [41].

In the following section, we will describe in detail the SCGF approach at finite temperature and the LW formalism that we will use in our calculations of the entropy. The numerical results derived from this formalism will be divided in two different parts. Section III will be devoted to the microscopic results, and Sec. IV will describe the bulk TD properties of a correlated system of nucleons. Finally, a brief summary will be given in Sec. V.

\section{FORMALISM}

\section{A. The ladder approximation}

With the help of the single-particle propagator, we can obtain all the one-body (and even some two-body) properties of a many-body system [42]. In the Green's function approach, one aims to compute the single-particle propagator, which, in the grand-canonical ensemble, is defined according to

$$
i G\left(\mathbf{k} t, \mathbf{k}^{\prime} t^{\prime}\right)=\operatorname{Tr}\left\{\hat{\rho} \mathcal{T}\left[a_{\mathbf{k}}(t) a_{\mathbf{k}^{\prime}}^{\dagger}\left(t^{\prime}\right)\right]\right\},
$$

where we have introduced the density matrix operator

$$
\hat{\rho}=\frac{1}{Z} e^{-\beta(\hat{H}-\mu \hat{N})}
$$

and the partition function

$$
Z=\operatorname{Tr}\left\{e^{-\beta(\hat{H}-\mu \hat{N})}\right\} .
$$

In Eq. (1), $\mathcal{T}$ is the time-ordering operator in such a way that the Heisenberg creation (annihilation) operator $a_{\mathbf{k}}^{\dagger}(t)\left[a_{\mathbf{k}}(t)\right]$ with the largest time argument $t$ (or $i t$ if $t$ is imaginary) is put to the left, with a minus sign included for each commutation. In these equations $\beta$ denotes the inverse temperature and $\mu$ is the chemical potential of the system. For simplicity, we will neglect the spin-isospin structure of this propagator in the following. Finally, the traces $\operatorname{Tr}$ are to be taken over all the energy and particle number eigenstates of the system. The cyclic invariance of these traces imply the following quasiperiodicity condition for the Green's function:

$$
G\left(\mathbf{k} t=0, \mathbf{k}^{\prime} t^{\prime}\right)=-e^{\beta \mu} G\left(\mathbf{k} t=-i \beta, \mathbf{k}^{\prime} t^{\prime}\right) .
$$

One can thus Fourier transform the time dependence of the propagator in terms of some Fourier coefficients $G\left(k, z_{v}\right)$, depending on the discrete Matsubara frequencies $z_{v}=\frac{(2 v+1) \pi}{-i \beta}+$ $\mu$. A Lehmann decomposition of these coefficients is achieved by means of the spectral function $\mathcal{A}(k, \omega)$ :

$$
G\left(k, z_{v}\right)=\int_{-\infty}^{\infty} \frac{d \omega}{2 \pi} \frac{\mathcal{A}(k, \omega)}{z_{v}-\omega} .
$$

The function $G\left(k, z_{v}\right)$ evaluated at the Matsubara frequencies can be analytically continued for all nonreal $z$. By using the Plemelj formula, the spectral function can be related with the values of $G$ close to the real axis:

$$
\mathcal{A}(k, \omega)=-2 \operatorname{Im} G\left(k, \omega_{+}\right)
$$

(where we use the notation $\omega_{+}=\omega+i \eta$ ). The single-particle Green's function can be obtained from Dyson's equation, which for a translationally invariant system reduces to the algebraic equation

$$
\left[\omega-\frac{k^{2}}{2 m}-\Sigma(k, \omega)\right] G(k, \omega)=1,
$$


where $\Sigma(k, \omega)$ denotes a complex self-energy. By expanding the self-energy in terms of one-particle Green's function, it can be shown that $\Sigma$ and $G$ share the same analytic properties. Thus, one writes the following spectral decomposition for $\Sigma$ :

$$
\Sigma(k, z)=\Sigma^{\mathrm{HF}}(k)+\int_{-\infty}^{\infty} \frac{d \omega}{2 \pi} \frac{\Gamma(k, \omega)}{z-\omega},
$$

where the width $\Gamma(k, \omega)$ is related to the imaginary part of $\Sigma$ by

$$
\Gamma(k, \omega)=-2 \operatorname{Im} \Sigma\left(k, \omega_{+}\right),
$$

and it is real and positive for all energy and momenta [43]. The term $\Sigma^{\mathrm{HF}}(k)$ in Eq. (8) is a real energy-independent generalized Hartree-Fock contribution to the self-energy.

The self-energy can be derived from an in-medium twobody interaction (the so-called scattering $T$ matrix) that includes the correlations induced by the strong short-range and tensor components of a realistic two-body $N N$ force. Within the ladder approximation, one can indeed relate the self-energy and the retarded $T$ matrix by $[44,45]$

$$
\begin{aligned}
\operatorname{Im} \Sigma\left(k, \omega_{+}\right)= & \int \frac{d^{3} k^{\prime}}{(2 \pi)^{3}} \int_{-\infty}^{\infty} \frac{d \omega^{\prime}}{2 \pi}\left\langle\mathbf{k} \mathbf{k}^{\prime}\left|\operatorname{Im} T\left(\omega+\omega_{+}^{\prime}\right)\right| \mathbf{k} \mathbf{k}^{\prime}\right\rangle_{A} \\
& \times \mathcal{A}\left(k^{\prime}, \omega^{\prime}\right)\left[f\left(\omega^{\prime}\right)+b\left(\omega+\omega^{\prime}\right)\right],
\end{aligned}
$$

where we have introduced the Fermi-Dirac distribution

$$
f(\omega)=\frac{1}{e^{\beta[\omega-\mu]}+1}
$$

and the Bose-Einstein distribution

$$
b(\Omega)=\frac{1}{e^{\beta[\Omega-2 \mu]}-1} .
$$

The pole of the Bose function $b(\Omega)$ at $\Omega=2 \mu$ is exactly canceled by a zero in the $T$ matrix [27,46] and thus the integrand remains finite as long as the $T$ matrix does not acquire a pole at this energy. This pole appears only for temperatures below a certain critical temperature and it is closely related to the onset of pairing among nucleons $[27,47]$.

Within the ladder approximation, the in-medium $T$ matrix is determined as a solution of the integral equation

$$
\begin{aligned}
\left\langle\mathbf{k} \mathbf{k}^{\prime}\left|T\left(\Omega_{+}\right)\right| \mathbf{p} \mathbf{p}^{\prime}\right\rangle_{A}= & \left\langle\mathbf{k} \mathbf{k}^{\prime}|V| \mathbf{p} \mathbf{p}^{\prime}\right\rangle_{A} \\
& +\int \frac{d^{3} q}{(2 \pi)^{3}} \int \frac{d^{3} q^{\prime}}{(2 \pi)^{3}}\left\langle\mathbf{k} \mathbf{k}^{\prime}|V| \mathbf{q} \mathbf{q}^{\prime}\right\rangle_{A} \\
& \times G_{I I}^{0}\left(\mathbf{q} \mathbf{q}^{\prime}, \Omega_{+}\right)\left\langle\mathbf{q} \mathbf{q}^{\prime}\left|T\left(\Omega_{+}\right)\right| \mathbf{p} \mathbf{p}^{\prime}\right\rangle_{A},
\end{aligned}
$$

where we have introduced the two-particle Green's function of two noninteracting but dressed nucleons:

$$
\begin{aligned}
G_{I I}^{0}\left(k_{1}, k_{2}, \Omega_{+}\right)= & \int_{-\infty}^{\infty} \frac{d \omega}{2 \pi} \int_{-\infty}^{\infty} \frac{d \omega^{\prime}}{2 \pi} \mathcal{A}\left(k_{1}, \omega\right) \mathcal{A}\left(k_{2}, \omega^{\prime}\right) \\
& \times \frac{1-f(\omega)-f\left(\omega^{\prime}\right)}{\Omega_{+}-\omega-\omega^{\prime}}
\end{aligned}
$$

Diagrammatically, the $T$-matrix approximation is depicted in Fig. 1, where the dressed one-particle propagators are given by two double lines. In general, the equations of motion arising within the Green's function method couple the $N$-particle propagator to the $(N+1)$-particle propagator whenever we have a two-body interaction. The Dyson equation for the

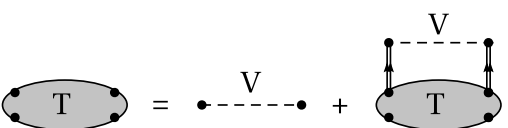

FIG. 1. Diagrammatical representation of the $T$ matrix within the ladder approximation.

single-particle Green's function involves, for instance, the full two-particle Green's function $G_{I I}$. In the SCGF approach within the ladder approximation, however, one ignores the effects of three- and more particle propagators by using a function, $G_{I I}^{0}$, which is a product of two dressed one-body propagators. In that way, one solves self-consistently the equations for the one-body $G$ and the approximated two-body $G_{I I}^{0}$ propagators.

If one wants to solve Eq. (13) in an efficient way, a partial wave decomposition is needed. After expressing $G_{I I}^{0}$ as a function of the total $\mathbf{P}=\mathbf{k}_{\mathbf{1}}+\mathbf{k}_{\mathbf{2}}$ and the relative momenta $\mathbf{k}_{\mathbf{r}}=\left(\mathbf{k}_{\mathbf{1}}-\mathbf{k}_{\mathbf{2}}\right) / 2$, one can perform the usual angle-average approximation on the angle between $\mathbf{P}$ and $\mathbf{k}_{\mathbf{r}}$, so that $G_{I I}^{0}$ is expressed only in terms of the modulus $P$ and $k_{r}$ :

$$
\begin{aligned}
\bar{G}_{I I}^{0}\left(P, k_{r}, \Omega_{+}\right)= & \frac{1}{2} \int_{-1}^{1} d(\cos \theta) \\
& \times G_{I I}^{0}\left(\left|\mathbf{P}+\mathbf{k}_{\mathbf{r}}\right|,\left|\mathbf{P}-\mathbf{k}_{\mathbf{r}}\right|, \Omega_{+}\right) .
\end{aligned}
$$

This approximation leads to a decoupling of the partial waves with different total angular momentum $J$, which in its turn implies that Eq. (13) becomes a one-dimensional integral equation:

$$
\begin{aligned}
\left\langle k_{r}\left|T_{L L^{\prime}}^{J S T}\left(P, \Omega_{+}\right)\right| k_{r}^{\prime}\right\rangle_{A}= & \left\langle k_{r}\left|V_{L L^{\prime}}^{J S T}\right| k_{r}^{\prime}\right\rangle_{A} \\
& +\sum_{L^{\prime \prime}} \int_{0}^{\infty} \frac{d k_{r}^{\prime \prime}}{(2 \pi)^{3}} k_{r}^{\prime \prime 2}\left\langle k_{r}\left|V_{L L^{\prime \prime}}^{J S T}\right| k_{r}^{\prime \prime}\right\rangle_{A} \\
& \times \bar{G}_{I I}^{0}\left(P, k_{r}^{\prime \prime}, \Omega_{+}\right) \\
& \times\left\langle k_{r}^{\prime \prime}\left|T_{L L^{\prime \prime}}^{J S T}\left(P, \Omega_{+}\right)\right| k_{r}^{\prime}\right\rangle_{A}
\end{aligned}
$$

By summing over all partial waves, we get the $T$ matrix as needed in the solution of Eq. (13):

$$
\begin{aligned}
\left\langle\mathbf{k k}^{\prime}\left|\operatorname{Im} T\left(\Omega_{+}\right)\right| \mathbf{k} \mathbf{k}^{\prime}\right\rangle_{A}= & \frac{1}{4 \pi} \sum_{J S T L}(2 J+1)(2 T+1) \\
& \times\left\langle q\left|\operatorname{Im} T_{L L}^{J S T}\left(P, \Omega_{+}\right)\right| q\right\rangle_{A}
\end{aligned}
$$

The only remaining piece is now the generalized Hartree-Fock contribution to the self-energy:

$$
\begin{aligned}
\Sigma^{\mathrm{HF}}(k)= & \frac{1}{8 \pi} \sum_{J S T L}(2 J+1)(2 T+1) \\
& \times \int \frac{d^{3} k^{\prime}}{(2 \pi)^{3}}\left\langle q\left(\mathbf{k}, \mathbf{k}^{\prime}\right)\left|V_{L L}^{J S T}\right| q\left(\mathbf{k}, \mathbf{k}^{\prime}\right)\right\rangle_{A} n\left(k^{\prime}\right),
\end{aligned}
$$

where we have introduced the momentum distribution

$$
n(k)=\int_{-\infty}^{\infty} \frac{d \omega}{2 \pi} \mathcal{A}(k, \omega) f(\omega) .
$$

In a self-consistent procedure, Eqs. (8), (10), and (13)-(16) are solved at a given temperature and density. The chemical 
potential $\mu$ is determined at each iteration by inverting

$$
\rho=v \int \frac{d^{3} k}{(2 \pi)^{3}} n(k, \mu),
$$

where $v$ denotes the spin-isospin degeneracy of the system $(v=4$ in the case of symmetric nuclear matter). As a final output, once convergence is reached, one obtains the singleparticle propagator from Dyson's equation and, from its imaginary part, the spectral function $\mathcal{A}(k, \omega)$. From this function, several micro- and macroscopic properties of the system can be computed. For instance, the momentum distribution given by Eq. (19) or the total energy per particle of the system, accessible from the GMK sum rule, is

$$
\frac{E^{\mathrm{GMK}}}{A}=\frac{v}{\rho} \int \frac{d^{3} k}{(2 \pi)^{3}} \int_{-\infty}^{\infty} \frac{d \omega}{2 \pi} \frac{1}{2}\left(\frac{k^{2}}{2 m}+\omega\right) \mathcal{A}(k, \omega) f(\omega),
$$

where $A$ is the total number of particles of the system. Further on, we will assess the question of how to compute the partition function and the entropy of a system of nucleons from the spectral function $\mathcal{A}$.

Before doing so, however, let us consider some interesting approximations to the SCGF method. By using Dyson's equation, it is straightforward to show that the spectral function can be written as

$$
\mathcal{A}(k, \omega)=\frac{\Gamma(k, \omega)}{\left[\omega-\frac{k^{2}}{2 m}-\operatorname{Re} \Sigma(k, \omega)\right]^{2}+\left[\frac{\Gamma(k, \omega)}{2}\right]^{2}} .
$$

It is interesting to note that this is a positive defined function for all energy and momenta [43], fulfilling the following sum rule [48-50]:

$$
\int_{-\infty}^{\infty} \frac{d \omega}{2 \pi} \mathcal{A}(k, \omega)=1
$$

One can obtain a simplified set of equations by taking the zerowidth limit, $\Gamma \rightarrow 0$ in the previous expression, thus obtaining the $\delta$ function:

$$
\mathcal{A}(k, \omega)=2 \pi \delta\left[\omega-\varepsilon_{\mathrm{qp}}(k)\right],
$$

where the quasiparticle spectrum is derived by means of the real part of the self-energy:

$$
\varepsilon_{\mathrm{qp}}(k)=\frac{k^{2}}{2 m}+\operatorname{Re} \Sigma\left[k, \varepsilon_{\mathrm{qp}}(k)\right] .
$$

This is the so-called quasiparticle self-consistent Green's Function method. In the following, we will not use this approximation. However, we shall extensively use the $\delta$-peak approximation to the spectral function, Eq. (24), with the single-particle energies $\varepsilon_{\mathrm{qp}}(k)$ defined by Eq. (25) with the self-energy of our SCGF calculation.

In addition, we will also consider the BHF approach to nuclear matter. This can be derived from the SCGF method if one takes the quasiparticle approximation, Eq. (24), and, in addition, one makes the following substitution:

$$
\left[1-f(\omega)-f\left(\omega^{\prime}\right)\right] \rightarrow[1-f(\omega)]\left[1-f\left(\omega^{\prime}\right)\right]
$$

in the two-particle propagator, Eq. (14). In the $T \rightarrow 0$ limit, this expression becomes the Pauli operator and $G_{I I}^{0}$ reduces to the particle-particle propagator. From this approximation, one can see that the $T$ matrix reduces to the well-known $G$-matrix in-medium interaction:

$$
\begin{aligned}
\left\langle\mathbf{k} \mathbf{k}^{\prime}\left|G\left(\Omega_{+}\right)\right| \mathbf{p} \mathbf{p}^{\prime}\right\rangle_{A}= & \left\langle\mathbf{k} \mathbf{k}^{\prime}|V| \mathbf{p} \mathbf{p}^{\prime}\right\rangle_{A} \\
& +\int \frac{d^{3} q}{(2 \pi)^{3}} \int \frac{d^{3} q^{\prime}}{(2 \pi)^{3}}\left\langle\mathbf{k} \mathbf{k}^{\prime}|V| \mathbf{q q}^{\prime}\right\rangle_{A} \\
& \times \frac{\left[1-f\left[\varepsilon_{\mathrm{BHF}}(q)\right]\right]\left[1-f\left[\varepsilon_{\mathrm{BHF}}\left(q^{\prime}\right)\right]\right]}{\Omega_{+}-\varepsilon_{\mathrm{BHF}}(q)-\varepsilon_{\mathrm{BHF}}\left(q^{\prime}\right)} \\
& \times\left\langle\mathbf{q q} \mathbf{q}^{\prime}\left|G\left(\Omega_{+}\right)\right| \mathbf{p} \mathbf{p}^{\prime}\right\rangle_{A},
\end{aligned}
$$

where the single-particle spectra of nucleons in the BHF approach, $\varepsilon_{\mathrm{BHF}}(k)$, are given by

$$
\varepsilon_{\mathrm{BHF}}(k)=\frac{k^{2}}{2 m}+\operatorname{Re} \Sigma_{\mathrm{BHF}}(k) .
$$

In this approximation, the self-energy is given in terms of the $G$ matrix by

$$
\begin{aligned}
\Sigma_{\mathrm{BHF}}(k)= & \int \frac{\mathrm{d}^{3} k^{\prime}}{(2 \pi)^{3}} f\left[\varepsilon_{\mathrm{BHF}}\left(k^{\prime}\right)\right] \\
& \times\left\langle\mathbf{k k}^{\prime}\left|G\left[\varepsilon_{\mathrm{BHF}}(k)+\varepsilon_{\mathrm{BHF}}\left(k^{\prime}\right)\right]\right| \mathbf{k k}^{\prime}\right\rangle_{A}
\end{aligned}
$$

and the total energy per particle is obtained from

$\frac{E^{\mathrm{BHF}}}{A}=\frac{\nu}{\rho} \int \frac{d^{3} k}{(2 \pi)^{3}}\left[\frac{k^{2}}{2 m}+\frac{1}{2} \operatorname{Re} \Sigma_{\mathrm{BHF}}(k)\right] f\left[\varepsilon_{\mathrm{BHF}}(k)\right]$.

We shall make two more comments concerning this finitetemperature generalization of the BHF approach. On the one hand, one must be aware that hole-hole propagation is the cause of the Bose term in Eq. (10). If the BHF is to be derived from the SCGF approach, one should neglect this term, so that the imaginary part of the self-energy reads

$$
\begin{aligned}
\operatorname{Im} \Sigma_{\mathrm{BHF}}(k)= & \int \frac{\mathrm{d}^{3} k^{\prime}}{(2 \pi)^{3}} f\left[\varepsilon_{\mathrm{BHF}}\left(k^{\prime}\right)\right] \\
& \times\left\langle\mathbf{k} \mathbf{k}^{\prime}\left|\operatorname{Im} G\left[\varepsilon_{\mathrm{BHF}}(k)+\varepsilon_{\mathrm{BHF}}\left(k^{\prime}\right)\right]\right| \mathbf{k k}^{\prime}\right\rangle_{A}
\end{aligned}
$$

On the other hand, one should say that the BHF approach obtained from this approximation of the SCGF method is not fully justified from basic first principles. Indeed, the finitetemperature generalization of the $T=0$ Bethe-Goldstone expansion is given by the $\mathrm{BdD}$ approach. In that theory, a similar expression to Eq. (27) for the in-medium temperaturedependent interaction can be obtained, but in principle there is not a straightforward relation with Eq. (30) for the energy per particle of the finite-temperature system. Nevertheless, one can see that this approximation is a reasonable one, because the dominant diagrams of the $\mathrm{BdD}$ expansion reduce to this finite-temperature BHF approach at low temperatures [21]. In the BHF approach that we will use here, the free energy is obtained from the energy per particle of Eq. (30) together with the mean-field expression for the entropy of the system:

$$
\begin{aligned}
S^{\mathrm{BHF}}= & -v \int \frac{d^{3} k}{(2 \pi)^{3}}\left\{f\left[\varepsilon_{\mathrm{BHF}}(k)\right] \ln f\left[\varepsilon_{\mathrm{BHF}}(k)\right]\right. \\
& \left.+\left(1-f\left[\varepsilon_{\mathrm{BHF}}(k)\right]\right) \ln \left(1-f\left[\varepsilon_{\mathrm{BHF}}(k)\right]\right)\right\} .
\end{aligned}
$$




\section{B. Luttinger-Ward formalism}

From a statistical mechanics point of view, the macroscopic information of the system is contained solely in the partition function. Once this function is known, its derivatives give access to the TD properties of the system. However, from a microscopic point of view, it is well known that the one-body properties can be derived from the dressed single-particle propagator of the system. Now one may ask whether there is a connection between both functions and, in particular, if the single-particle propagator is enough for building the partition function. The answer, given by Luttinger and Ward [39] more than forty years ago, is yes. The expression for the partition function thus obtained turns out to have some interesting properties that were later on exploited by Baym [51] in his well-known discussion of the TD consistency of many-body approaches. The starting point is the so-called Luttinger-Ward expression of the partition function of the system:

$$
\ln Z=\operatorname{Tr} \Sigma\left(k, z_{v}\right) G\left(k, z_{v}\right)+\operatorname{Tr} \ln \left[-G^{-1}\left(k, z_{v}\right)\right]-\Phi[G],
$$

where the functional $\Phi[G]$ has been introduced and where the trace $\mathrm{Tr}$ means a sum over all momentum states and Matsubara frequencies:

$$
\operatorname{Tr} \rightarrow \sum_{\vec{k}, v} e^{z_{\nu} \eta}
$$

with $\eta=0^{+}$small and positive and such that $\lim _{\operatorname{Re} z \rightarrow \infty} \eta \operatorname{Re} z=\infty$. In TD equilibrium, the grand-partition function is stationary under variations of the Green's function,

$$
\left.\frac{\delta \ln Z}{\delta G}\right|_{G_{0}}=0,
$$

and thus the functional $\Phi$ should satisfy the following condition:

$$
\left.\frac{\delta \Phi}{\delta G}\right|_{G_{0}}=\Sigma .
$$

To obtain the previous expression, we have used Dyson's equation, Eq. (7), to functionally derive the self-energy $\Sigma$ with respect to $G$. In fact, within the LW formalism, $\Sigma$ is a functional of the propagator and one can take Eq. (36) as its definition. Usually, however, in a given many-body approach one assumes a certain approximation to the self-energy (in our case, the ladder approximation). The functional $\Phi$ is then fixed by Eq. (36) and the propagator is given by Dyson's equation, $G^{-1}=G_{0}^{-1}+\Sigma$. These ideas can be depicted diagrammatically. For the ladder approximation, we show in Fig. 2 the $n$ th-order contribution to the self-energy $\Sigma^{(n)}$ (where $n$ denotes the number of bare interaction lines in the diagram). By closing this diagram in its free vertices with a propagator, we obtain the corresponding $n$ th-order contribution to $\Phi$. At each order, however, this contribution should be divided by a factor of $2 n$ that takes into account the $2 n$ possible places where one can cut each propagator to obtain $\Sigma$ from Eq. (36) [51]. The corresponding series for the functional is shown in terms of diagrams in Fig. 3.

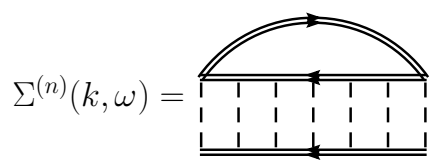

FIG. 2. $n$th order contribution to the ladder self-energy.

The evaluation of the sum in the partition function has to be done with special care because of the cut in the logarithm. The final expression for the grand potential $\Omega=-T \ln Z$ reads

$$
\begin{aligned}
\Omega= & v \int \frac{d^{3} k}{(2 \pi)^{3}} \int_{-\infty}^{\infty} \frac{d \omega}{2 \pi} f(\omega) 2 \operatorname{Im}\left\{\ln \left[-G^{-1}\left(k, \omega_{+}\right)\right]\right. \\
& \left.+\Sigma\left(k, \omega_{+}\right) G\left(k, \omega_{+}\right)\right\}+T \Phi[G] .
\end{aligned}
$$

In this expression, the propagator and the self-energy are computed above but close to the real axis. We have also introduced the Fermi-Dirac distribution $f(\omega)$, Eq. (11). One can now readily obtain the entropy by means of the TD relation

$$
S=-\left.\frac{\partial \Omega}{\partial T}\right|_{\mu} .
$$

The stationarity of $\Omega$ with respect to changes in $G$ is now very useful, because it implies that in Eq. (37) only the temperature derivatives of the Fermi functions are needed:

$$
\begin{aligned}
S= & v \int \frac{d^{3} k}{(2 \pi)^{3}} \int_{-\infty}^{\infty} \frac{d \omega}{2 \pi} \frac{\partial f(\omega)}{\partial T} 2 \operatorname{Im}\left\{\ln \left[-G^{-1}\left(k, \omega_{+}\right)\right]\right. \\
& \left.+\Sigma\left(k, \omega_{+}\right) G\left(k, \omega_{+}\right)\right\}+\frac{\partial}{\partial T} T \Phi[G] .
\end{aligned}
$$

This expression gives the entropy per unit volume of a correlated system of fermions as a function of $G, \Sigma$, and $\Phi$ and it is the fundamental equation from which we will derive most of our results. The usefulness and applications of this formula in the context of Fermi liquids were extensively discussed in the pioneering work of Carneiro and Pethick [6]. In the following, we will closely follow this reference and discuss some of the more relevant approximations for our case.

One can explicitly compute the imaginary parts of the two terms inside the integrals. The first term is the imaginary part of a logarithm (i.e., the phase of its argument). We can thus decompose the argument into its real and imaginary parts:

$$
\begin{aligned}
G^{-1}\left(k, \omega_{+}\right) & =\omega-\frac{k^{2}}{2 m}-\operatorname{Re} \Sigma(k, \omega)-i \operatorname{Im} \Sigma\left(k, \omega_{+}\right) \\
& =\operatorname{Re} G^{-1}(k, \omega)+\frac{i}{2} \Gamma(k, \omega) .
\end{aligned}
$$

We consider that the logarithm has a cut in the real negative axis and we work in the sheet where $\ln (1)=0$. The $\arctan (z)$ function goes from $-\pi / 2$ to $\pi / 2$ and is normally used to obtain the phase of a complex number. Nevertheless, whenever the

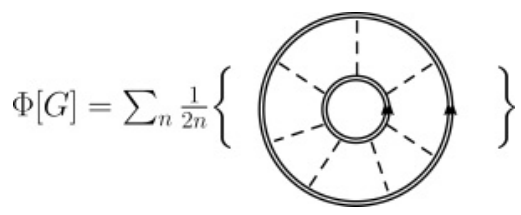

FIG. 3. $\Phi$ functional for the $T$-matrix approximation. 
real part of $G^{-1}$ becomes positive and provided that $\Gamma(k, \omega)$ is positive defined, the complex phase of the logarithm's argument will be in the quadrant III of the complex plane and thus a factor $\pi$ needs to be subtracted from the arctan function to match the argument of $-G^{-1}$. The result of the imaginary part can thus be recast in the following form:

$$
\begin{aligned}
\operatorname{Im}\left\{\ln \left[-G^{-1}\left(k, \omega_{+}\right)\right]\right\}= & \arctan \lambda(k, \omega)-\pi \theta \\
& \times\left[\operatorname{Re} G^{-1}(k, \omega)\right],
\end{aligned}
$$

where we have introduced the function

$$
\lambda(k, \omega)=\frac{\Gamma(k, \omega)}{2 \operatorname{Re} G^{-1}(k, \omega)},
$$

which is nothing but the quotient between the imaginary and the real part of the complex inverse propagator. The imaginary part of the second term in the integral of Eq. (39) is given by

$$
\begin{aligned}
\operatorname{Im}\left\{\Sigma\left(k, \omega_{+}\right) G\left(k, \omega_{+}\right)\right\}= & \operatorname{Re} \Sigma\left(k, \omega_{+}\right) \operatorname{Im} G\left(k, \omega_{+}\right) \\
& +\operatorname{Im} \Sigma\left(k, \omega_{+}\right) \operatorname{Re} G\left(k, \omega_{+}\right) \\
= & -\frac{1}{2} \operatorname{Re} \Sigma(k, \omega) \mathcal{A}(k, \omega) \\
& -\frac{1}{2} \Gamma(k, \omega) \operatorname{Re} G(k, \omega) .
\end{aligned}
$$

Using Eqs. (41) and (43), we can divide the entropy $S$ into two terms,

$$
S=S^{\mathrm{DQ}}+S^{\prime}
$$

given by

$$
S^{\mathrm{DQ}}=v \int \frac{d^{3} k}{(2 \pi)^{3}} \int_{-\infty}^{\infty} \frac{d \omega}{2 \pi} \frac{\partial f(\omega)}{\partial T} \Xi(k, \omega)
$$

and

$$
\begin{aligned}
S^{\prime}= & -\frac{\partial}{\partial T} T \Phi[G]+v \int \frac{d^{3} k}{(2 \pi)^{3}} \int_{-\infty}^{\infty} \frac{d \omega}{2 \pi} \frac{\partial f(\omega)}{\partial T} \\
& \times \mathcal{A}(k, \omega) \operatorname{Re} \Sigma(k, \omega),
\end{aligned}
$$

where we have introduced the function

$$
\begin{aligned}
\Xi(k, \omega)= & 2 \pi \theta\left[\operatorname{Re} G^{-1}(k, \omega)\right]-2 \arctan \lambda(k, \omega) \\
& +\Gamma(k, \omega) \operatorname{Re} G(k, \omega) .
\end{aligned}
$$

$S^{\mathrm{DQ}}$ is a dynamical quasiparticle (DQ) entropy, which partially takes into account the correlations of the dressed particles in the medium. It includes some finite-width effects, as seen by the fact that it is computed with a nonzero $\Gamma$. The term $S^{\prime}$ accounts for higher order correlations. As was shown in [6], this term arises from the cancellation between the temperature derivative of $T \Phi$ and the second term of Eq. (46). The only nonzero contributions that survive this cancellation come from terms in the perturbation expansion that have at least two vanishing energy denominators. In the following, we shall make the assumption that $S^{\prime}$ is negligible. In this way, our formalism is simplified because there is no need to evaluate the $\Phi$ functional. This assumption, of course, needs to be validated, and that is what we will do in the final part of this work. As we will see, our approximation leads to TD consistent results, which confirms that the contribution of $S^{\prime}$ is small in the density and temperature range explored as far as short-range correlations are concerned. In addition, it is worth mentioning that it is precisely from the $S^{\prime}$ contribution that all the anomalous temperature dependences of the entropy arise. These anomalies, however, are mainly generated by terms in $S^{\prime}$ involving long-range correlations, which we do not consider in our approach. Thus, by restricting ourselves to computing the entropy with $S^{\mathrm{DQ}}$, we will lose these contributions and only find analytical $\left(S \sim T, T^{3}\right)$ temperature dependences.

To study the DQ entropy, it is interesting to analyze the properties of the function $\Xi(k, \omega)$. The first term of $\Xi$ in Eq. (47) is a step function with argument $\operatorname{Re} G^{-1}$. For a fixed momentum $k$ and as a function of the energy, this argument is negative for energies below the quasiparticle peak $\varepsilon_{\mathrm{qp}}(k)$, whereas it is positive for $\omega$ greater than $\varepsilon_{\mathrm{qp}}(k)$. Thus the step function can be rewritten as

$\Xi_{1}(k, \omega)=2 \pi \theta\left[\omega-\frac{k^{2}}{2 m}-\operatorname{Re} \Sigma(k, \omega)\right]=2 \pi \theta\left[\omega-\varepsilon_{\mathrm{qp}}(k)\right]$.

At a fixed momentum, then, $\Xi_{1}$ equals zero at energies below the quasiparticle pole and $2 \pi$ above it. By using the relation

$$
\frac{\partial f(\omega)}{\partial T}=-\frac{\partial \sigma(\omega)}{\partial \omega},
$$

where we have introduced the function

$$
\sigma(\omega)=-\{f(\omega) \ln f(\omega)+[1-f(\omega)] \ln [1-f(\omega)]\},
$$

the contribution of $\Xi_{1}$ to the entropy is given by

$$
\begin{aligned}
S_{1}^{\mathrm{DQ}} & =v \int \frac{d^{3} k}{(2 \pi)^{3}} \int_{-\infty}^{\infty} \frac{d \omega}{2 \pi} \frac{\partial \sigma(\omega)}{\partial \omega} 2 \pi \theta\left[\omega-\varepsilon_{\mathrm{qp}}(k)\right] \\
& =v \int \frac{d^{3} k}{(2 \pi)^{3}} \int_{-\infty}^{\varepsilon_{\mathrm{qp}}(k)} d \omega \frac{\partial \sigma(\omega)}{\partial \omega} \\
& =v \int \frac{d^{3} k}{(2 \pi)^{3}} \sigma\left[\varepsilon_{\mathrm{qp}}(k)\right] \equiv S^{\mathrm{QP}} .
\end{aligned}
$$

This expression corresponds to the entropy of a system of undamped quasiparticles with real quasiparticle energies given by Eq. (25). Whenever quasiparticles have long lifetimes, we expect it to be a good approximation to the entropy. Indeed, for any many-body approximation where the quasiparticle energies are real (such as the Hartree-Fock case, for instance) the full DQ entropy is simply given by Eq. (51). The rest of the terms in $\Xi$ can be rewritten as a function of $\lambda(k, \omega)$ :

$$
\Xi_{2}(k, \omega)=-2 \arctan [\lambda(k, \omega)]
$$

for the second term and

$$
\Xi_{3}(k, \omega)=\frac{2 \lambda(k, \omega)}{1+\lambda^{2}(k, \omega)},
$$

for the third one. Their total contribution to the entropy is then given by

$$
\begin{aligned}
S_{2}^{D Q}= & v \int \frac{d^{3} k}{(2 \pi)^{3}} \int \frac{d \omega}{2 \pi} \frac{\partial f(\omega)}{\partial T}\left\{\frac{2 \lambda(k, \omega)}{1+\lambda^{2}(k, \omega)}\right. \\
& -2 \arctan [\lambda(k, \omega)]\} .
\end{aligned}
$$

This expression involves a nonvanishing width $\Gamma$ and it can thus be thought of as a lifetime correction to the DQ entropy. 
It is clear that for infinitely long lived quasiparticles $(\Gamma=0)$, this contribution will be zero, but for large widths it can have a non-negligible effect on the total entropy.

Let us return to Eq. (45) for the DQ entropy. After performing a partial integration and using relation (49), we see that the following expression for the DQ entropy holds:

$$
S^{\mathrm{DQ}}=v \int \frac{d^{3} k}{(2 \pi)^{3}} \int_{-\infty}^{\infty} \frac{d \omega}{2 \pi} \sigma(\omega) \mathcal{B}(k, \omega),
$$

provided that the $\mathcal{B}$ spectral function is defined as

$$
\mathcal{B}(k, \omega)=\frac{\partial \Xi(k, \omega)}{\partial \omega} .
$$

Equation (55) has several interesting properties. First, in the free and the Hartree-Fock cases the $\mathcal{B}$ function reduces to a delta peak and $S^{\mathrm{DQ}}$ becomes the expected expression for the entropy per particle. However, this does not mean that $S^{\mathrm{DQ}}$ neglects the finite width of quasiparticles, as we already commented. In addition, it is easy to check that the $\mathcal{B}$ function fulfils the following sum rule:

$$
\int_{-\infty}^{\infty} \frac{d \omega}{2 \pi} \mathcal{B}(k, \omega)=\left.\frac{1}{2 \pi} \Xi(k, \omega)\right|_{-\infty} ^{\infty}=1 .
$$

Finally, let us remark that this expression is somewhat intuitive, in the sense that it is a product of the statistical weighting factor for the entropy $\sigma(\omega)$ times a spectral function that takes into account the width of quasiparticles.

An alternative way to obtain the entropy within the LW formalism is obtained by starting from Eq. (37) and using the fact that

$$
\beta f(\omega)=-\frac{\partial}{\partial \omega} \ln \left[1+e^{-\beta(\omega-\mu)}\right] .
$$

Integrating by parts one easily obtains

$$
\begin{aligned}
\Omega= & -v \int \frac{d^{3} k}{(2 \pi)^{3}} \int_{-\infty}^{\infty} \frac{d \omega}{2 \pi} T \ln \left[1+e^{-\beta(\omega-\mu)}\right] B(k, \omega) \\
& +T \Phi[G],
\end{aligned}
$$

where we have introduced the function

$$
\begin{aligned}
B(k, \omega)= & -\frac{\partial}{\partial \omega} 2 \operatorname{Im}\left\{\ln \left[-G^{-1}\left(k, \omega_{+}\right)\right]\right. \\
& \left.+\Sigma\left(k, \omega_{+}\right) G\left(k, \omega_{+}\right)\right\} .
\end{aligned}
$$

The entropy is now obtained via the temperature derivative of Eq. (59), which will only affect the explicit temperature factors because of the stationarity condition, Eq. (35):

$$
S=v \int \frac{d^{3} k}{(2 \pi)^{3}} \int_{-\infty}^{\infty} \frac{d \omega}{2 \pi} \sigma(\omega) B(k, \omega)+\frac{\partial}{\partial T} T \Phi[G] .
$$

Different expressions for $B$ can be obtained depending on whether the derivative or the imaginary part is taken first. By taking first the imaginary part and then the derivative, we see that the following relation holds between $\Xi$ and $B$ :

$$
\begin{aligned}
B(k, \omega)= & \frac{\partial}{\partial \omega} \Xi(k, \omega)+\frac{\partial \operatorname{Re} \Sigma(k, \omega)}{\partial \omega} \mathcal{A}(k, \omega) \\
& +\operatorname{Re} \Sigma(k, \omega) \frac{\partial \mathcal{A}(k, \omega)}{\partial \omega} .
\end{aligned}
$$

For the first term of Eq. (60), however, it is instructive to proceed in the opposite direction; first differentiating and then taking the imaginary part, we obtain

$$
\begin{aligned}
B_{1}(k, \omega)= & -2 \operatorname{Im}\left\{\frac{\partial}{\partial \omega} \ln \left[-G^{-1}\left(k, \omega_{+}\right)\right]\right\} \\
= & -2 \operatorname{Im}\left\{G\left(k, \omega_{+}\right)\left[1-\frac{\partial \Sigma\left(k, \omega_{+}\right)}{\partial \omega}\right]\right\} \\
= & \mathcal{A}(k, \omega)\left[1-\frac{\partial \operatorname{Re} \Sigma(k, \omega)}{\partial \omega}\right] \\
& -\operatorname{Re} G(k, \omega) \frac{\partial \Gamma(k, \omega)}{\partial \omega} .
\end{aligned}
$$

The derivative of the second term is easily computed and can be separated into two parts:

$B_{2}(k, \omega)=\frac{\partial \operatorname{Re} G(k, \omega)}{\partial \omega} \Gamma(k, \omega)+\operatorname{Re} G(k, \omega) \frac{\partial \Gamma(k, \omega)}{\partial \omega}$

and

$$
B_{3}(k, \omega)=\frac{\partial \operatorname{Re} \Sigma(k, \omega)}{\partial \omega} \mathcal{A}(k, \omega)+\operatorname{Re} \Sigma(k, \omega) \frac{\partial \mathcal{A}(k, \omega)}{\partial \omega} .
$$

Now we can write a compact expression for the $B$ function:

$$
\begin{aligned}
B(k, \omega)= & \mathcal{A}(k, \omega)+\frac{\partial \mathcal{A}(k, \omega)}{\partial \omega} \operatorname{Re} \Sigma(k, \omega) \\
& +\frac{\partial \operatorname{Re} G(k, \omega)}{\partial \omega} \Gamma(k, \omega) .
\end{aligned}
$$

In addition, if one uses the fact that $B_{3}$ equals the last two terms of Eq. (62), it is easy to obtain the following relation between the $B$ and the $\mathcal{B}$ spectral functions:

$$
\begin{aligned}
\mathcal{B}(k, \omega)= & B_{1}(k, \omega)+B_{2}(k, \omega)=\mathcal{A}(k, \omega)\left[1-\frac{\partial \operatorname{Re} \Sigma(k, \omega)}{\partial \omega}\right] \\
& +\frac{\partial \operatorname{Re} G(k, \omega)}{\partial \omega} \Gamma(k, \omega),
\end{aligned}
$$

which also gives $\mathcal{B}$ as a function of $\mathcal{A}$ and the real and imaginary parts of the propagator.

The expression for the entropy of Eq. (61), in terms of the $\Phi$ functional plus a term containing the integral of a statistical factor $\sigma(\omega)$ and the weighting function $B(k, \omega)$, has already been obtained in, for instance, Ref. [40]. However, one should take into account that our approximation for the entropy, $S^{\mathrm{DQ}}$, differs from the first term of Eq. (61). In our case, we have neglected the terms of Eq. (46), which are in fact canceling each other to a certain degree, whereas by approximating the entropy with the term of the $B$ spectral function one would be ignoring this cancellation. In fact, there is no reason to believe that the first term of Eq. (61) should be a good approximation to the full entropy, while the DQ entropy, given by the convolution of the statistical factor $\sigma(\omega)$ and the $B$ spectral function, gives very reasonable results as we shall see in the following.

Also illustrative is the following decomposition of $S^{\mathrm{DQ}}$ into two terms:

$$
S_{1}^{A}=v \int \frac{d^{3} k}{(2 \pi)^{3}} \int_{-\infty}^{\infty} \frac{d \omega}{2 \pi} \sigma(\omega) \mathcal{A}(k, \omega)
$$


and

$$
\begin{aligned}
S_{2}^{A}= & v \int \frac{d^{3} k}{(2 \pi)^{3}} \int_{-\infty}^{\infty} \frac{d \omega}{2 \pi} \sigma(\omega)\left\{\frac{\partial \operatorname{Re} G(k, \omega)}{\partial \omega} \Gamma(k, \omega)\right. \\
& \left.-\mathcal{A}(k, \omega) \frac{\partial \operatorname{Re} \Sigma(k, \omega)}{\partial \omega}\right\} .
\end{aligned}
$$

This justifies somehow the naive generalization to the expression of the entropy, that has been used in the literature [52,53] and which consists in approximating the entropy by formula (68). This is, of course, not justified from TD grounds, but it would be a natural extension of Eq. (19) to the case of the entropy. In particular, within a quasiparticle approximation, when both $\mathcal{A}(k, \omega)$ and $\mathcal{B}(k, \omega)$ become quasiparticle delta functions, $S^{\mathrm{DQ}}$ and $S_{1}^{A}$ coincide and become the quasiparticle approximation to the entropy, $S^{\mathrm{QP}}$,

$$
S^{\mathrm{QP}}=v \int \frac{d^{3} k}{(2 \pi)^{3}} \sigma\left[\varepsilon_{\mathrm{qp}}(k)\right] .
$$

The DQ entropy, however, goes beyond the naive quasiparticle approach. It introduces the corrections of Eq. (69), which, as we will see later on, are non-negligible.

Another justification for Eq. (55) comes from the generalization of the $\Phi$-functional technique to nonequilibrium quantum systems. In Ref. [54], it was shown that, within certain $\Phi$-derivable approaches out of equilibrium, an $H$ theorem could be proved for a nonequilibrium kinetic entropy expressed in terms of the full Green's function and the self-energy. For equilibrium systems, this kinetic entropy reduces to the sum of $S^{\mathrm{DQ}}$, the local or Markovian part of the kinetic entropy ( $S_{\text {loc }}$ in the language of Ref. [54]), plus $S^{\prime}$, the memory or non-Markovian part of the entropy $\left(S_{\mathrm{mem}}\right)$, which coincides with the expression of the entropy Eq. (44).

If $S^{\mathrm{DQ}}$ is close to the real entropy of the correlated system $S$, we should recover TD consistency (i.e., the microscopically computed quantities should coincide with the macroscopically computed ones). The ladder approximation is known to be a well-defined $\Phi$-derivable approach [51]. In particular, the (microscopic) chemical potential $\tilde{\mu}$ computed from the normalization condition

$$
\rho=v \int \frac{d^{3} k}{(2 \pi)^{3}} \int_{-\infty}^{\infty} \frac{d \omega}{2 \pi} \mathcal{A}(k, \omega) f(\omega, \tilde{\mu})
$$

should coincide with the (macroscopic) chemical potential coming from the TD expression

$$
\mu(\rho, T)=\left.\frac{\partial F(\rho, T)}{\partial \rho}\right|_{T},
$$

with $F$ the free-energy per unit volume. At $T=0$, this has been numerically checked for the SCGF with separable potentials in [55]. However, it is also well known that some many-body approximations do not fulfill this check of consistency. The BHF approach, for instance, badly violates the Hugenholtz-van Hove theorem [56], which states that, at saturation density, the chemical potential $\tilde{\mu}$ and the free energy per particle $F / A$ should coincide. The difference between these two quantities can be as large as $20 \mathrm{MeV}$ [57].
In the following, we will present the numerical results for the $\mathcal{B}$ spectral function and the different approximations to the entropy obtained from SCGF calculations. Our aim is twofold. On the one hand, we will show that the computation of the DQ entropy is enough to maintain TD consistency; that is, that for the range of densities and temperatures considered $S^{\mathrm{DQ}}$ gives a free energy that respects the Hugenholtz-van Hove theorem. This consistency is embedded in the ladder approximation (as shown by Baym [51]), but it is lacking in other many-body approaches. In addition, the TD consistency of our results seems to indicate that the $S^{\prime}$ contribution to the entropy is not crucial in the nuclear matter case. Note that an exact evaluation of $S^{\prime}$ would require knowledge of the $\Phi$ functional. This functional has recently been computed also within a ladder approximation [40], in a SCGF computation that differs from ours only in some numerical details. The results that we will present (specially those concerning the temperature dependence of the entropy) agree substantially with those of Ref. [40]. We believe that this is an indication of the consistency of both approaches.

\section{MICROSCOPIC RESULTS}

All the results quoted in this and the following sections have been obtained with the finite-temperature SCGF approach of Ref. [33] using the CDBONN potential [58]. In the numerical treatment, partial waves up to $J=8$ have been included. The Born approximation has been used for $J \geqslant 3$. The quoted BHF results have been computed with the same $N N$ potential with partial waves up to $J=4$. None of the calculations includes three-body forces. We are thus unable to reproduce the saturation point of SNM. In this sense, the results presented here should be taken as a first study of the TD properties within SCGF theory, focused on the effects that correlations induce on the entropy of SNM.

In the previous section we have mentioned that the properties of the $\mathcal{B}$ spectral function are very close to those of the usual spectral function $\mathcal{A}(k, \omega)$. It fulfils a sum rule and it accounts somehow for the effect of the width of quasiparticles in the DQ entropy. It is thus natural to compare the two functions on the same plot. We can get a rough idea of the differences of both functions following an argument first proposed by Carneiro and Pethick. Let us express the spectral function $\mathcal{A}$ as a function of the real and imaginary parts of the self-energy. We will of course obtain the well-known Lorentzian-like function of Eq. (22). For a given momentum, the spectral function will have a peak around the quasiparticle energy of height $\mathcal{A} \sim 4 / \Gamma\left[k, \varepsilon_{\mathrm{qp}}(k)\right]$. However, the $\mathcal{B}$ spectral function can also be rewritten in terms of the self-energy. Starting from Eq. (47) and taking the derivative with respect to the energy, we get

$$
\begin{aligned}
\mathcal{B}(k, \omega)= & \frac{1}{2} \frac{\Gamma^{3}(k, \omega)}{\left[\left[\omega-\frac{k^{2}}{2 m}-\operatorname{Re} \Sigma(k, \omega)\right]^{2}+\left[\frac{\Gamma(k, \omega)}{2}\right]^{2}\right]^{2}} \\
& \times\left\{1-\frac{\partial \operatorname{Re} \Sigma(k, \omega)}{\partial \omega}\right\}
\end{aligned}
$$




$$
\begin{aligned}
& -\frac{1}{2} \frac{\Gamma^{2}(k, \omega)}{\left[\left[\omega-\frac{k^{2}}{2 m}-\operatorname{Re} \Sigma(k, \omega)\right]^{2}+\left[\frac{\Gamma(k, \omega)}{2}\right]^{2}\right]^{2}} \\
& \times\left\{\omega-\frac{k^{2}}{2 m}-\operatorname{Re} \Sigma(k, \omega)\right\} \frac{\partial \Gamma(k, \omega)}{\partial \omega} .
\end{aligned}
$$

If one assumes that the frequency dependence of $\Gamma$ and $\operatorname{Re} \Sigma$ are smooth close to the quasiparticle energy, we will have

$$
\mathcal{B}(k, \omega) \sim \frac{1}{2} \frac{\Gamma^{3}(k, \omega)}{\left[\left[\omega-\frac{k^{2}}{2 m}-\operatorname{Re} \Sigma(k, \omega)\right]^{2}+\left[\frac{\Gamma(k, \omega)}{2}\right]^{2}\right]^{2}},
$$

which corresponds to a function that decays faster than a Lorentzian close to $\varepsilon_{\mathrm{qp}}(k)$, but which has a stronger peak at the quasiparticle energy, $\mathcal{B} \sim 8 / \Gamma\left[k, \varepsilon_{\mathrm{qp}}(k)\right]$.

One can check that this schematic scenario is true in Fig. 4, where we show the $\mathcal{B}$ (full lines) and the $\mathcal{A}$ (dashed lines) spectral functions as a function of the energy at three different momenta $k=0, k=k_{F}$, and $k=2 k_{F}$, at the experimental saturation density $\rho=0.16 \mathrm{fm}^{-3}$ and at a temperature of $T=$ $10 \mathrm{MeV}$. In all three panels, corresponding to the three different

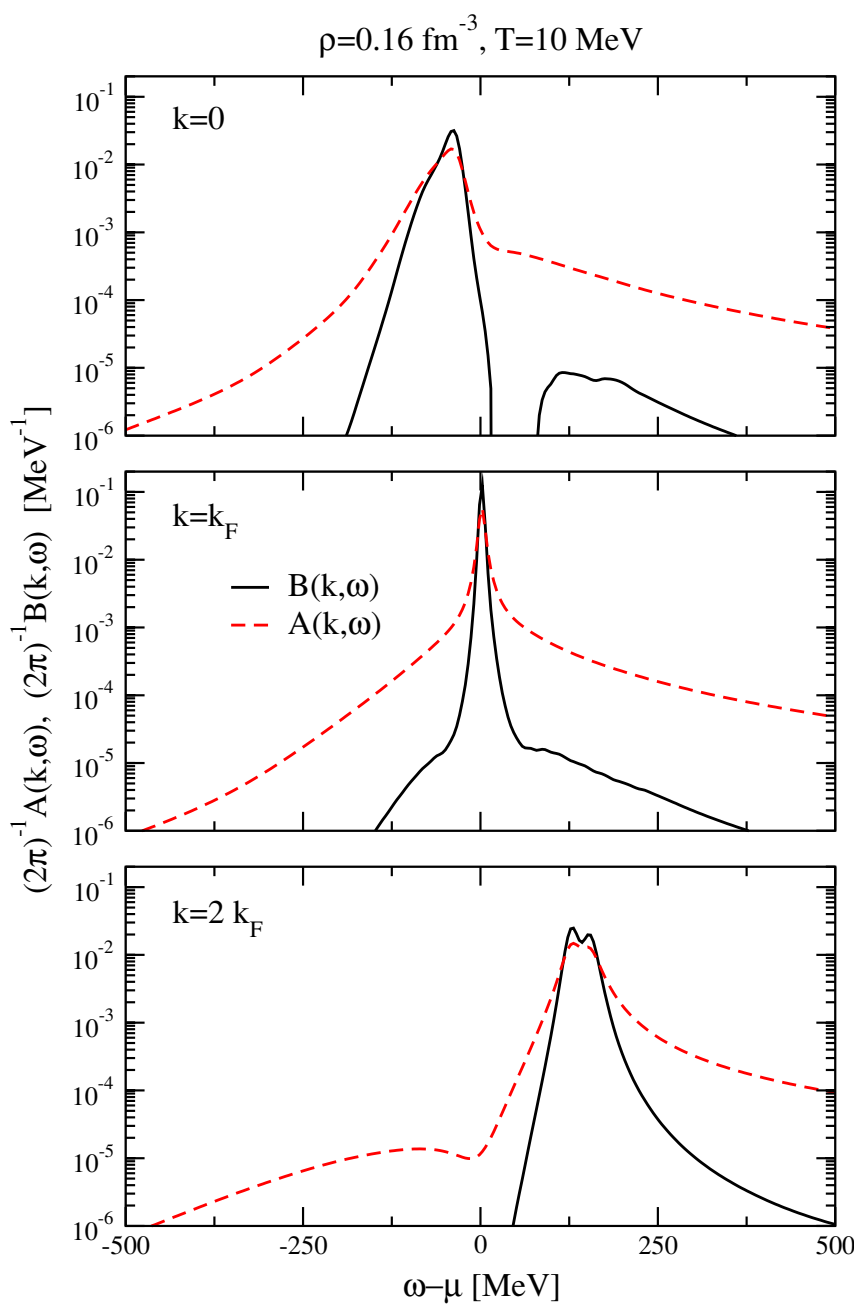

FIG. 4. (Color online) $\mathcal{B}$ (solid lines) and $\mathcal{A}$ (dashed lines) spectral functions at $\rho=0.16 \mathrm{fm}^{-3}$ and $T=10 \mathrm{MeV}$ for three different momenta $k=0, k_{F}$, and $2 k_{F}$. momenta, we see that both functions are peaked around the same energy values, corresponding to the quasiparticle energies given by Eq. (25). The peaks shift from negative values (with respect to the chemical potential) to positive values when going from zero-momentum to higher momentum states, just following the position of the quasiparticle peak. However, although the $\mathcal{A}$ spectral function has high-energy tails that contribute in a non-negligible way to the total strength of the nucleon, the $\mathcal{B}$ spectral function has lower and less extended energy tails. This is easily understood if one considers that both functions fulfill the same sum rule. Since the $\mathcal{B}$ function has a higher quasiparticle peak, the strength of the peak is contributing substantially to the total sum rule and there is no need to generate high-energy tails. The presence of these high-energy tails in the $\mathcal{A}$ function is an indication of the importance of the correlations that go beyond the mean-field approach [33]. Thus, the lack of such tails in the $\mathcal{B}$ function signals that these correlations will have a small influence on the total entropy of the system.

This idea is also in accordance with the behavior of the width of both spectral functions. Far away from the Fermi momenta, both functions are relatively broad around the peak. Again, in the case of the $\mathcal{A}$ function this is a consequence of the correlations that redistribute the nucleon single-particle strength within a wide range of energies. The $\mathcal{B}$ function has a smaller width, which indicates that it is less affected by correlations. Close to the Fermi momentum, however, both functions approach a delta-peak behavior, reminiscent of the fact that at zero temperature, even when correlations are included, the spectral function has a delta-peak contribution. At this momentum and for the temperature considered, the $\mathcal{B}$ function is narrower and much more peaked than the usual spectral function $\mathcal{A}$.

Also note that the values for the $\mathcal{B}$ function are positive for all the energies and momenta that we have considered in our investigation. This contrasts with the weighting function $B$, which is defined in Eq. (60) and has been used in Ref. [40]. The fact that the evaluation of the entropy using the weighting function $B$ exhibits strong cancellation effects (see Fig. 4 of Ref. [40]) may be taken as an indication that the splitting of the entropy into the two contributions according to Eq. (61) might not be optimal.

To understand the density dependence of the DQ entropy, we show in Fig. 5 the $\mathcal{B}$ spectral function as a function of the energy for different densities $\left(\rho=0.1,0.2,0.3,0.4,0.5 \mathrm{fm}^{-3}\right)$ at the same three different momenta previously compared and at a fixed temperature of $T=10 \mathrm{MeV}$. In addition, we plot with a dotted line the statistical weighting function $\sigma(\omega)$. It is precisely the product of these two functions, integrated over energies and momenta, that gives rise to the DQ entropy, so it is interesting to study their overlap.

The general features of the $\mathcal{B}$ spectral function as a function of density are very close to those of the usual spectral function $\mathcal{A}$. In the case of $k=0$, the quasiparticle peak moves to more and more attractive energies as density increases, reflecting the fact that the binding energy of a zero-momentum nucleon increases with density. Above the Fermi surface (at $k=2 k_{F}$ ), the situation is the opposite and the peak of the $\mathcal{B}$ function moves to higher energies with increasing density. The width 

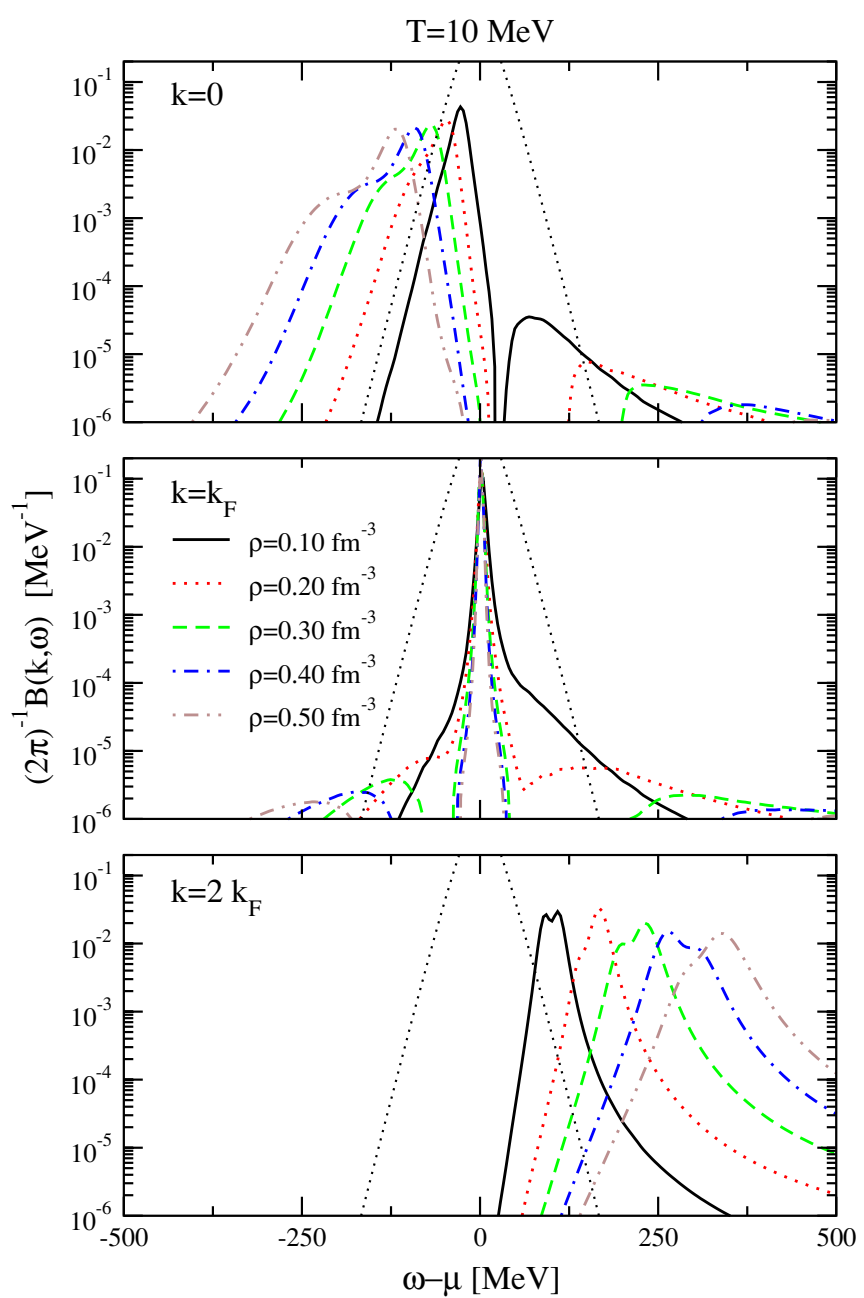

FIG. 5. (Color online) $\mathcal{B}$ spectral function for five different densities (from $\rho=0.1$ to $\rho=0.5 \mathrm{fm}^{-3}$ in equidistant steps) at a fixed temperature of $T=10 \mathrm{MeV}$ and three different momenta $k=0, k_{F}$, and $2 k_{F}$.

of these peaks, both at zero momentum and at twice the Fermi momentum, is broadened with density. This is in accordance with the naive idea that correlation effects increase with density. In addition, as a consequence of this broadening, the high-energy tails (visible at high positive energies for the $k=0$ state) decrease with density, allowing the sum rule [Eq. (57)] to be fulfilled.

The situation is different at the Fermi surface: When the density is increased, the peak remains at a fixed energy $\omega=\mu$, whereas its width becomes narrower and concentrates more strength. This can be understood if one takes into account that, as already commented, at zero temperature (i.e., for the fully degenerate system), the correlated $\mathcal{A}$ spectral function shows a delta peak that would also be present for the $\mathcal{B}$ spectral function. At a fixed nonzero temperature, however, the system moves toward the degenerate limit (the ratio $T / \epsilon_{F}$ decreases) with increasing density and thus the $\mathcal{B}$ spectral function becomes closer to a delta peak. This is actually what can be seen in the central panel of Fig. 5. At high densities $\left(\rho \geqslant 0.2 \mathrm{fm}^{-3}\right)$, a clear separation between the quasiparticle peak and the background contribution to the $\mathcal{B}$ spectral function is observed.

It is clear from Fig. 5 that the quasiparticle peak and the peak of the $\sigma$ function only coincide for momenta close to $k_{F}$ and energies around $\omega=\mu$. Thus, the important contributions to the DQ entropy of the system will be that of the momenta close to the Fermi surface and the energies close to the chemical potential. It is precisely the interplay between $\sigma$ and $\mathcal{B}$ that gives rise to the density dependence of the entropy. Since the value of $\mathcal{B}$ at $k=k_{F}$ and $\omega=\mu$ increases with density, one may expect that the entropy per particle would increase with density. However, it is also true that, for lower densities, the quasiparticle peak is closer to $\mu$ at all momenta and thus there are contributions of the quasiparticle peak for momenta not necessarily close to $k_{F}$. In fact, when these contributions are summed, one finds that the entropy per particle decreases with density.

To illustrate these results, we plot in Fig. 6 the momentumdependent integrand of Eq. (55):

$$
\zeta(k)=\frac{v}{2 \pi^{2}} k^{2} \int_{-\infty}^{\infty} \frac{d \omega}{2 \pi} \sigma(\omega) \mathcal{B}(k, \omega),
$$

which measures the contribution to the DQ entropy from each momentum state. It is clear that, as density increases, the integrand becomes larger at the Fermi surface but less extended in momenta. This is in agreement with the previously discussed ideas, that is, that for less degenerate systems the contributions at all momenta are relevant, whereas for degenerate systems the contribution of the $k=k_{F}$ state is the most important one. In addition, the circles in the figure show the $\zeta$ function obtained within a quasiparticle approximation:

$$
\zeta^{\mathrm{QP}}(k)=\frac{v}{2 \pi^{2}} k^{2} \sigma\left[\varepsilon_{\mathrm{qp}}(k)\right]
$$

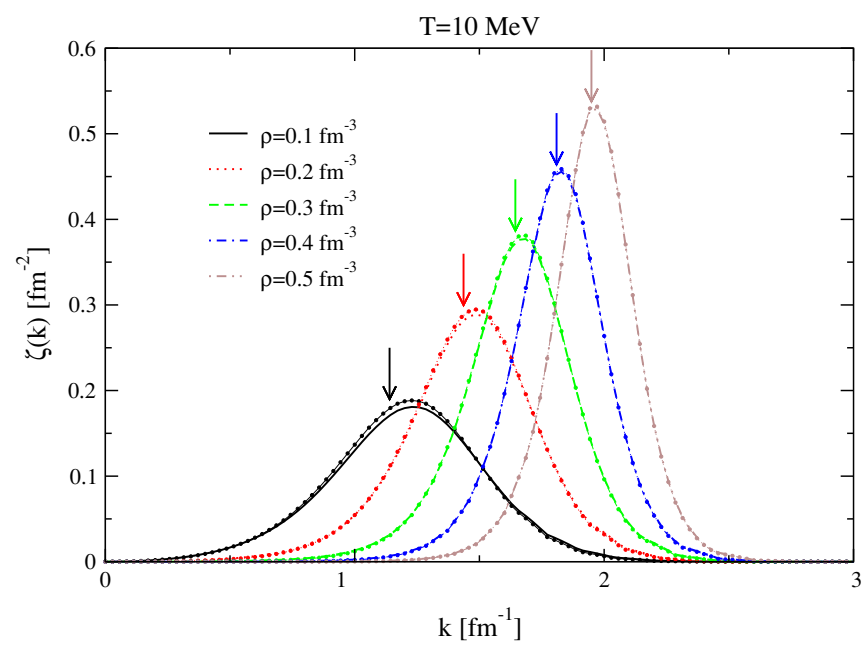

FIG. 6. (Color online) Momentum dependence of the $\zeta$ function [see Eq. (75)] for five different densities (from $\rho=0.1$ to $\rho=$ $0.5 \mathrm{fm}^{-3}$ in equidistant steps) at a fixed temperature of $T=10 \mathrm{MeV}$. The dots correspond to the quasiparticle approximation $\zeta^{\mathrm{QP}}$ for the same densities and temperature. The arrows signal the position of the Fermi momentum at each density. 
with the quasiparticle peak given by the maximum of the spectral function, Eq. (25). The differences are only relevant for the lowest densities and in a range of momenta close to the Fermi momentum. This is again a signature of the small role played by the correlations that deplete the quasiparticle states on the entropy. Therefore, we expect that the quasiparticle approximation to the entropy, Eq. (70), will describe the full DQ entropy $S^{\mathrm{DQ}}$ quite well.

To gain insight into the temperature dependence of the DQ entropy, we show in Fig. 7 the $\mathcal{B}$ spectral function as a function of energy for the same three different momenta considered previously at a fixed density $\rho=0.16 \mathrm{fm}^{-3}$ and at five different temperatures $T=4,8,12,16,20 \mathrm{MeV}$. It is clear that, for all momenta, the variations of temperature mainly result in changes of the width of the quasiparticle peak, whereas the position in energy of this peak relative to the chemical potential is nearly unchanged. In addition, the momentum states far above the Fermi surface are not affected by temperature, as is seen in the lowest panel, corresponding to $k=2 k_{F}$. At the Fermi surface, however, the effects are much more important.
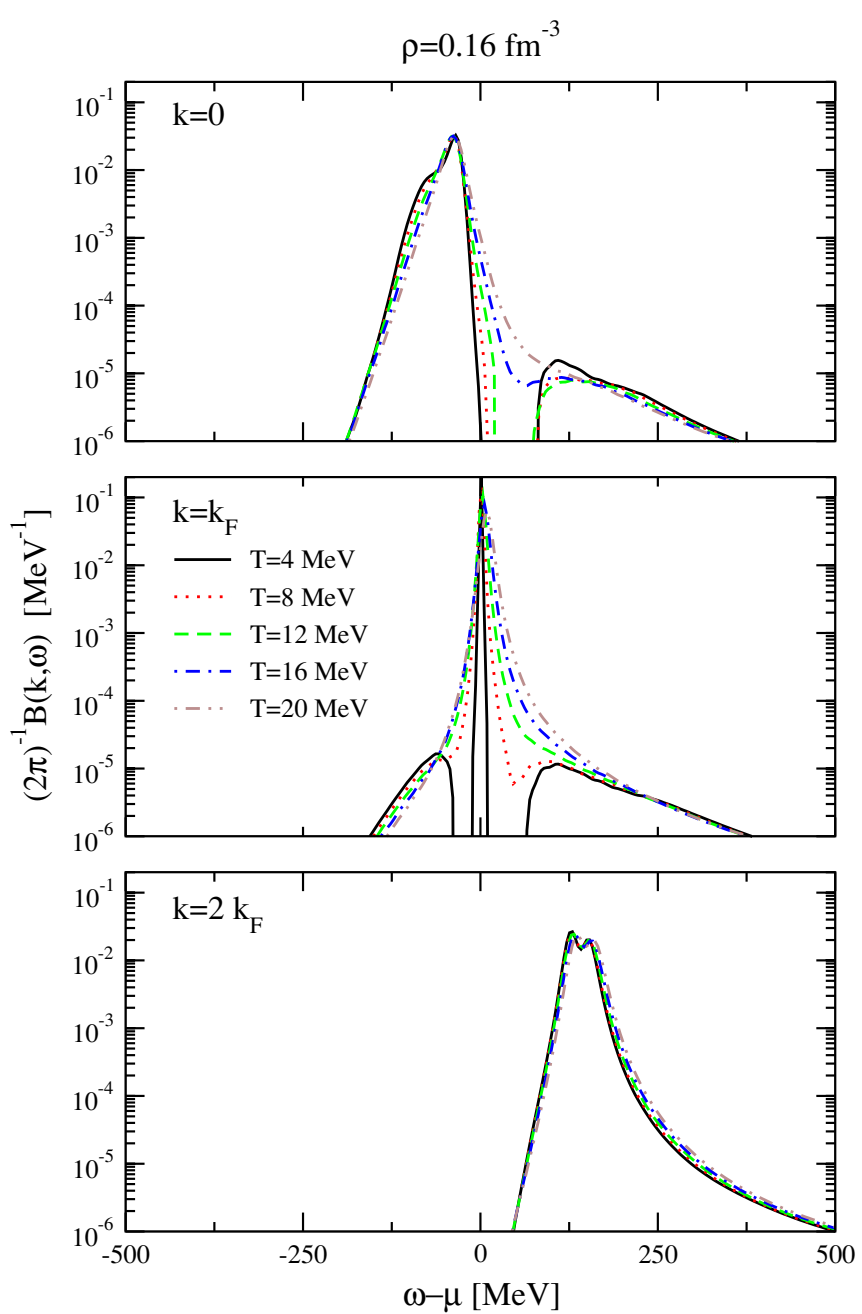

FIG. 7. (Color online) $\mathcal{B}$ spectral function for five different temperatures (from $T=4$ to $T=20 \mathrm{MeV}$ in equidistant steps) at a fixed density of $\rho=0.16 \mathrm{fm}^{-3}$ and three different momenta $k=0, k_{F}$, and $2 k_{F}$.
As temperature increases, the height of the quasiparticle peak decreases, whereas its width increases. Moreover, at the lowest temperature $(T=4 \mathrm{MeV})$ a clean separation is observed between a quasiparticle peak and a particle and hole background. This separation is softened at $T=8 \mathrm{MeV}$ and disappears completely above this temperature. Such a behavior is again understood in terms of the degeneracy of the system: The lower the temperature, the higher the degeneracy and the smaller the width of the $\mathcal{B}$ spectral function. For the $k=0$ state, a similar situation is found. The peak lies below the chemical potential, and it is clearly split from the particle background at $T=$ $4 \mathrm{MeV}$. For temperatures above $T=12 \mathrm{MeV}$ this separation disappears and a smooth transition from particle to hole states is found in the $\mathcal{B}$ function. It is also interesting to notice that the width of the peak remains more or less constant, thus indicating that temperature-induced effects on the width of the quasiparticle peak are more important at $k=k_{F}$.

For the total contribution to the DQ entropy, the convolution between $\sigma(\omega)$ and $\mathcal{B}(k, \omega)$ is again crucial. At low temperatures, $\sigma(\omega)$ is very peaked around $\omega \sim \mu$. The convolution will thus only be different from zero whenever the quasiparticle peak is close to $\mu$ (i.e., at $k \sim k_{F}$ ). However, at higher temperatures $\sigma(\omega)$ differs from zero in a wider region of energies, which results in a nonzero convolution at all momenta. When we integrate over momenta, the final DQ entropy is higher for the higher temperature. Thus, in accordance with intuition, the entropy of this correlated system will grow with temperature.

\section{MACROSCOPIC RESULTS}

In this section we will explore the density and temperature dependences of the entropy computed within different approximations. To begin, we show in Fig. 8 the density dependence of the entropy per particle at a fixed temperature $T=10 \mathrm{MeV}$. The approximations to the entropy per particle that appear in this figure are as follows:

(i) $S^{\mathrm{DQ}}$, the full dynamical quasiparticle entropy of Eq. (45) and equivalently Eq. (55) (solid lines);

(ii) $S^{\mathrm{QP}}$, the quasiparticle approximation to the entropy of Eq. (70) (dotted lines);

(iii) $S^{\mathrm{BHF}}$, the Brueckner-Hartree-Fock entropy computed from expression Eq. (32) (dashed lines); and

(iv) $S_{1}^{A}$, the contribution to the entropy from the $\mathcal{A}$ spectral function (dash-dotted line).

As a general feature, we can say that all of these entropies decrease substantially with density, from values of around 2.5 at densities of around $0.02 \mathrm{fm}^{-3}$ down to values of around 0.4 for the highest density considered here, $\rho=0.5 \mathrm{fm}^{-3}$.

One important result that arises from Fig. 8 is the fact that, at all densities, $S^{\mathrm{DQ}}$ and $S^{\mathrm{QP}}$ are very close. This is somehow in agreement with the idea that the inclusion of the width of quasiparticles has a small effect on the entropy. As discussed in relation with Fig. 6, the effect is larger at lower densities, where both approximations differ more, but it is never higher than $5 \%$. At high densities, the difference is so small that it cannot be appreciated in the figure. This result is not at 


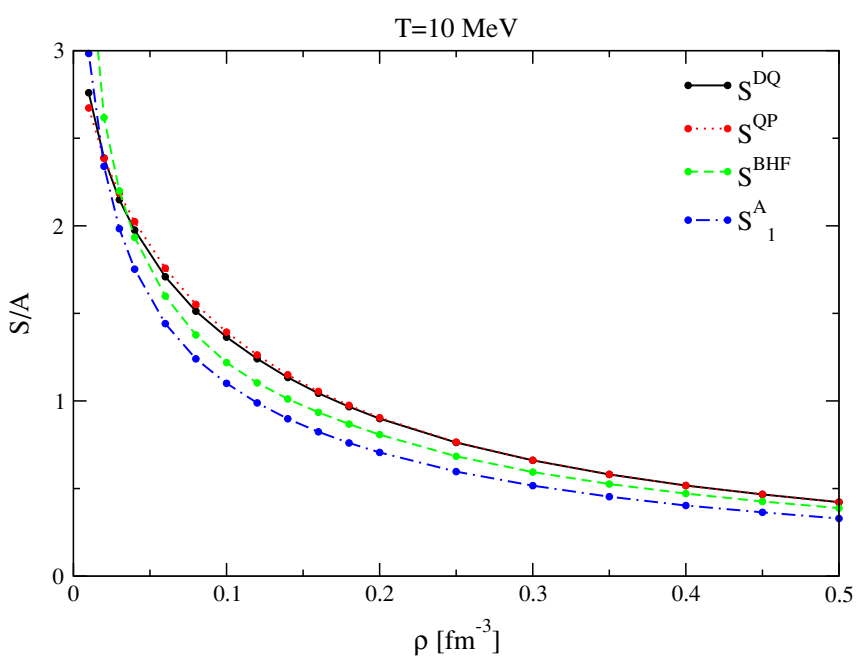

FIG. 8. (Color online) Different approximations to the entropy as a function of density for a $T=10 \mathrm{MeV}$ temperature. The full lines correspond to $S^{\mathrm{DQ}}$, the dotted lines to $S^{\mathrm{QP}}$, the dashed lines to $S^{\mathrm{BHF}}$, and the dot-dashed lines to $S_{1}^{A}$.

all intuitive. It indicates that $S_{2}^{\mathrm{DQ}}$, which is nothing but the difference between $S^{\mathrm{DQ}}$ and $S^{\mathrm{QP}}$, decreases with density. But, since we have argued that $S_{2}^{\mathrm{DQ}}$ represents somehow the finite width effects on the entropy and since correlations grow with density, we would also expect it to grow with density. However, we have also seen that the higher the density, the smaller the width of the $\mathcal{B}$ spectral function at $k=k_{F}$ (which is the more relevant contribution at high densities) and thus the lower the effects of correlations. This is why at higher densities both approximations to the entropy tend to be similar. The fact that $S_{2}^{\mathrm{DQ}}$ is negative at intermediate densities (say from $\rho=$ $0.05 \mathrm{fm}^{-3}$ to $0.30 \mathrm{fm}^{-3}$ ) is quite interesting: In addition to stressing the fact that finite-lifetime effects to the entropy are small, one can say that it looks like correlations (i.e., the width of the quasiparticle) tend to order the system.

The effects of the self-consistent propagation of holes are responsible for the difference between $S^{\mathrm{DQ}}$ and $S^{\mathrm{BHF}}$. This can be clearly seen by rewriting Eq. (32) to give

$$
S^{\mathrm{BHF}}=v \int \frac{d^{3} k}{(2 \pi)^{3}} \sigma\left[\varepsilon_{\mathrm{BHF}}(k)\right],
$$

which differs from Eq. (70) in the position of the single-particle peaks [given by $\varepsilon_{\mathrm{qp}}(k)$ in the first case and by $\varepsilon_{\mathrm{BHF}}(k)$ in the second] and also in the different values of the chemical potentials in the statistical factor $\sigma$. Since the effect of the width on $S^{\mathrm{DQ}}$ is small at this temperature, the difference between both entropies arises from the different quasiparticle energies and chemical potentials of the two approaches. Each of these quantities can differ by at most $20 \mathrm{MeV}$ [33]. However, the correction in the entropy is small probably because of a cancellation between both differences in the argument of $\sigma$, where $\varepsilon(k)$ and $\mu$ are subtracted. In the intermediate-density region, the BHF entropy has values that are about $10 \%$ below the DQ one. The presence of hole-hole correlation thus increases the entropy (i.e., the thermal disorder). This is of course related to the fact that hole-hole correlations tend to increase the density of single-particle states close to the Fermi energy. If one tries to parametrize the quasiparticle spectrum close to $\mu$ in terms of an effective mass $m^{*}$, one obtains larger values for the parametrization of the SCGF spectrum than for BHF [33].

Finally, we also show the contribution of $S_{1}^{A}$ to the DQ entropy. As we have already mentioned, this expression comes from a naive generalization to incorporate width effects, but nevertheless it gives a reasonable first guess to the entropy per particle. Intuitively, one would expect that, since the $\mathcal{A}$ spectral function is wider than the $\mathcal{B}$ one, the overlap between $\mathcal{A}(k, \omega)$ and $\sigma(\omega)$ at a given momentum should be higher and the final $S_{1}^{A}$ entropy would overestimate $S^{\mathrm{DQ}}$. However, this is not the case, except for the lowest densities. This can be understood from the height of the quasiparticle peak for $\mathcal{A}$ being, roughly speaking, a factor of 2 lower than that for $\mathcal{B}$. Thus, although more extended in momentum, the $\zeta(k)$ function for the $\mathcal{A}$ spectral function is smaller and gives rise to a lower entropy. The difference of both entropies is between 20 and $30 \%$ for the intermediate-density region. The origin of such differences is the $S_{2}^{A}$ contribution of Eq. (69), which is the integral of two terms. Both terms are of the same order at $\rho \sim 0.1 \mathrm{fm}^{-3}$ but, although the contribution proportional to $\mathcal{A}$ decreases with density, the one proportional to $\Gamma$ increases, and above $\rho=0.3 \mathrm{fm}^{-3}$ it carries more than $80 \%$ of the total correction.

In Fig. 9 we show the temperature dependence of the entropy for a density $\rho=0.16 \mathrm{fm}^{-3}$ computed with the same approximations as just discussed. In addition, we have also computed another approximation, $S^{N K}$, which is displayed with a double-dotted dashed line. This corresponds to the entropy of the correlated momentum distribution, Eq. (19), obtained from the mean-field-like expression:

$S^{N K}=-v \int \frac{d^{3} k}{(2 \pi)^{3}}\{n(k) \ln n(k)+[1-n(k)] \ln [1-n(k)]\}$.

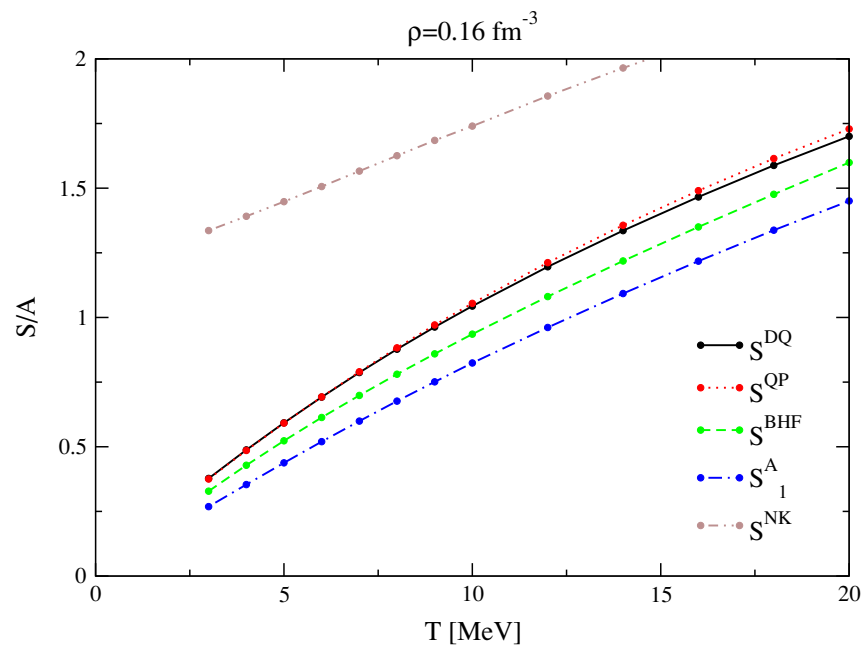

FIG. 9. (Color online) Different approximations to the entropy as a function of temperature at a density of $\rho=0.16 \mathrm{fm}^{-3}$. The full lines correspond to $S^{\mathrm{DQ}}$, the dotted lines to $S^{\mathrm{QP}}$, the dashed lines to $S^{\mathrm{BHF}}$, the dot-dashed lines to $S_{1}^{A}$, and the double dot-dashed lines to $S^{N K}$. 
One can say that all the approximations to the entropy of Fig. 9 decrease with temperature (as expected) and approach a linear dependence at low $T$. It is a well-known feature of Fermi liquids that the slope coefficient for such a linear behavior is proportional to the zero-temperature density of states computed at the Fermi surface, $N(T=0)$ :

$$
\frac{S_{\text {low }}}{A}=\frac{\pi^{2}}{3 \rho} N(0) T .
$$

Each of our approximations goes to the $T=0$ limit with different slopes, and we can thus obtain different densities of states. To calculate $N(0)$ for each approximation, however, we should extrapolate our results to the $T=0$ limit, which we cannot do within our approach reliably. Instead, from a low-temperature expansion of Eqs. (55) and (68) we obtain an expression of $N(0)$ in terms of the $\mathcal{B}$ and $\mathcal{A}$ spectral functions that we extend to finite temperatures. Namely, we get the "density of states" related to the $\mathcal{B}$ spectral function,

$$
N_{\mathcal{B}}(T)=v \int \frac{d^{3} k}{(2 \pi)^{4}} \mathcal{B}(k, \omega=\mu),
$$

and the usual one, related to the $\mathcal{A}$ spectral function,

$$
N_{\mathcal{A}}(T)=v \int \frac{d^{3} k}{(2 \pi)^{4}} \mathcal{A}(k, \omega=\mu),
$$

where $T$ denotes that these have been computed at the finite temperature at which the spectral functions have been computed. Note that at low $T$ and $\omega=\mu$ the functions $\mathcal{A}$ and $\mathcal{B}$ differ basically by a factor $\mathcal{Z}$ (where $\mathcal{Z}$ denotes the renormalization of the quasiparticle pole):

$$
\mathcal{A}(k, \omega=\mu) \sim \mathcal{Z}^{-1}(k, \omega=\mu) \mathcal{B}(k, \omega=\mu),
$$

and thus the two densities of states will also differ by such a factor. Nevertheless, the density of states that gives the correct linear fit to the DQ entropy is that of Eq. (80). In fact, we have numerically checked that it is this quantity that reduces to the well-known quasiparticle expression

$$
N_{\mathrm{QP}}(0)=\frac{\nu k_{F} m^{*}\left(k_{F}\right)}{2 \pi^{2} \hbar^{2}}
$$

at low enough temperatures, where the effective mass is obtained through the derivative

$$
\frac{m^{*}}{m}=\frac{1}{2 m}\left(\frac{d \varepsilon_{\mathrm{qp}}(k)}{d k^{2}}\right)^{-1}
$$

evaluated at the Fermi surface using the finite-temperature SCGF quasiparticle spectrum, $\varepsilon_{\mathrm{qp}}(k)$. This can be seen in Table I, where we give the densities of states computed with the $\mathcal{A}$ and the $\mathcal{B}$ spectral functions together with that obtained from the QP expression, Eq. (83), at $\rho=0.16 \mathrm{fm}^{-3}$ for low temperatures. The effective mass at $k_{F}$ is given in the fifth column of Table I. The numerical values confirm that at low temperatures the density of states from the $\mathcal{B}$ spectral function reduces to the quasiparticle one, hence indicating that from a TD point of view this is the correct density of states. However, from a microscopic point of view, the $\mathcal{A}$ density of states is the one that has been commonly used $[59,60]$. One should keep in mind that in a mean-field approximation both of them would
TABLE I. Densities of states related to the $\mathcal{A}$ (first column) and $\mathcal{B}$ (second column) spectral functions at $\rho=0.16 \mathrm{fm}^{-3}$ for different temperatures. The quasiparticle approximation to the density of states, Eq. (83), is displayed in the fourth column, together with the effective mass at the Fermi surface in the fifth column.

\begin{tabular}{ccccc}
\hline \hline$T(\mathrm{MeV})$ & $\begin{array}{c}N_{\mathcal{A}}(T) \\
\left(\mathrm{MeV}^{-1} \mathrm{fm}^{-3}\right)\end{array}$ & $\begin{array}{c}N_{\mathcal{B}}(T) \\
\left(\mathrm{MeV}^{-1} \mathrm{fm}^{-3}\right)\end{array}$ & $\begin{array}{c}N_{\mathrm{QP}}(T) \\
\left(\mathrm{MeV}^{-1} \mathrm{fm}^{-3}\right)\end{array}$ & $m^{*} / m$ \\
\hline 4 & 0.00435 & 0.00608 & 0.00608 & 0.935 \\
6 & 0.00430 & 0.00585 & 0.00586 & 0.901 \\
8 & 0.00424 & 0.00566 & 0.00570 & 0.875 \\
10 & 0.00416 & 0.00548 & 0.00557 & 0.855 \\
\hline \hline
\end{tabular}

reduce to the same expression, Eq. (83). It is also interesting to note that if we compute the entropy with the help of the "densities of states" coming from the $\mathcal{B}$ spectral function, Eq. (80), and from the QP expression, Eq. (83) (both of them being evaluated at the corresponding finite temperature), and we use them instead of $N(0)$ in Eq. (79), we can reproduce the DQ expression of the entropy for temperatures up to $T=$ $10 \mathrm{MeV}$ with less than a $10 \%$ discrepancy.

In the context of nucleus-nucleus collisions at intermediate energies, there exists a growing amount of experimental data $[61,62]$ that should be useful to constrain the thermal properties of nuclei and nuclear matter. In particular, the liquid-gas phase transition and the caloric curve give a hint on the properties of nuclei at low temperatures. In the study of the caloric curve, it is customary to parametrize the excitation energy at low temperatures in terms of the so-called inverse level density parameter $K$ (see [63] for a theoretical description), which is inversely proportional to the density of states introduced here. The values we obtain for $K$ [defined as $K^{-1}=\frac{\pi^{2}}{6} N_{\mathcal{B}}(0)$ ] are close to the Fermi gas value $K \sim 14.6 \mathrm{MeV}$ for $\rho=$ $0.16 \mathrm{fm}^{-3}$. This can be understood from the fact that $N_{\mathcal{B}}$ reduces to the quasiparticle value of Eq. (83), which, in addition, is similar to the free Fermi gas value because in our case the effective mass is almost equal to the bare-nucleon mass at low temperatures. A word of caution must be raised, however. Our value for $K$ is obtained from a calculation in infinite isospin symmetric matter in which only short-range correlations are treated. Nevertheless, it is clear that a study of the inverse level parameter should include both the effects of finite size and long-range correlations, which are very important in determining the low-energy excitations of nuclei.

In the following, we compare the different approximations to the entropy that appear in Fig. 9. As a general trend, we observe the same features that we have already discussed when we have commented the density dependence of the various approximations to the entropy. The quasiparticle approximation $S^{\mathrm{QP}}$ using SCGF energies reproduces the DQ entropies at all temperatures very well, especially below $T=10 \mathrm{MeV}$. The finite-temperature BHF entropy describes the entropy of the system, with an error of about $15 \%$. This difference is thus quite small, which is again a signal that both the depletion of single-particle strength and the exact position of the quasiparticle peak are not that crucial in the final result of the entropy per particle. Let us also note that 
the inclusion of the hole-hole propagation in the quasiparticle peaks tends to increase the entropy.

The $\mathcal{A}$ spectral function contribution to the entropy is again correct in the temperature behavior, but still it gives a too small value for the entropy per particle of the system, with errors as large as a $30 \%$. As we have previously discussed, this is due to the lower quasiparticle peak of the $\mathcal{A}$ spectral function, which makes the $S_{1}^{A}$ contribution to the entropy lower than the $S^{\mathrm{DQ}}$ entropy. The difference between these two functions is given by the $S_{2}^{A}$ contribution to the entropy. This is composed of the two terms in Eq. (69), which have different relative weights as temperature changes. Whereas the term proportional to the $\mathcal{A}$ spectral function amounts to $90 \%$ of the total correction at $T=4 \mathrm{MeV}$, its relative importance decreases linearly to a $30 \%$ contribution for the $T=20 \mathrm{MeV}$ case.

Finally, the entropy of Eq. (78) is given in terms of the fully correlated momentum distributions. This momentum distribution includes both thermal effects (which are capital for any entropy computation) plus correlation effects. In fact, in the final entropy $S^{N K}$ both effects are taken as the same and correlations somehow mimic extra thermal effects. This is why this is the only approximation that tends to give a nonzero entropy at $T=0$. In the fully degenerate limit, the momentum distributions given by Eq. (19) are not Fermi step functions and they are corrected by correlation effects. These correlations are responsible for a certain amount of entropy, when this is computed with Eq. (78) at $T=0$. Thus, at finite temperatures, thermal effects are overestimated in $S^{N K}$ owing to the presence of correlations, and $S^{N K}$ produces far too large an entropy (by almost a factor of 3 at $T=5 \mathrm{MeV}$ ).

After having computed the entropy, we would like to address the subject of TD consistency. From first principles, the ladder approximation is known to be $\Phi$-derivable [51]. Indeed, we have computed an expression for the entropy within a formalism that preserves $\Phi$-derivability. Thus, whenever $S^{\prime}$ is negligible in our approach, we expect our results to preserve TD consistency. Figure 10 shows the accuracy that we reach with our SCGF results. For the sake of comparison, we also show the BHF results. The upper solid lines with full circles correspond to the free energies per particle computed within the SCGF approach:

$$
F^{\mathrm{SCGF}}=E^{\mathrm{GMK}}-T S^{\mathrm{DQ}},
$$

with the energy computed with the GMK sum rule, Eq. (21), and the entropy with the DQ expression, Eq. (45). The BHF free energy is shown in a full line with solid diamonds and is simply given by

$$
F^{\mathrm{BHF}}=E^{\mathrm{BHF}}-T S^{\mathrm{BHF}},
$$

with the energy computed from the generalization of the $T=0$ BHF approach, Eq. (30), and the entropy from Eq. (32). The dotted lines with empty circles and diamonds correspond to the microscopic chemical potentials $\tilde{\mu}$ obtained from inverting Eq. (71) for the SCGF and from inverting

$$
\rho=v \int \frac{d^{3} k}{(2 \pi)^{3}} f\left(\varepsilon_{\mathrm{BHF}}(k), \tilde{\mu}^{\mathrm{BHF}}\right)
$$

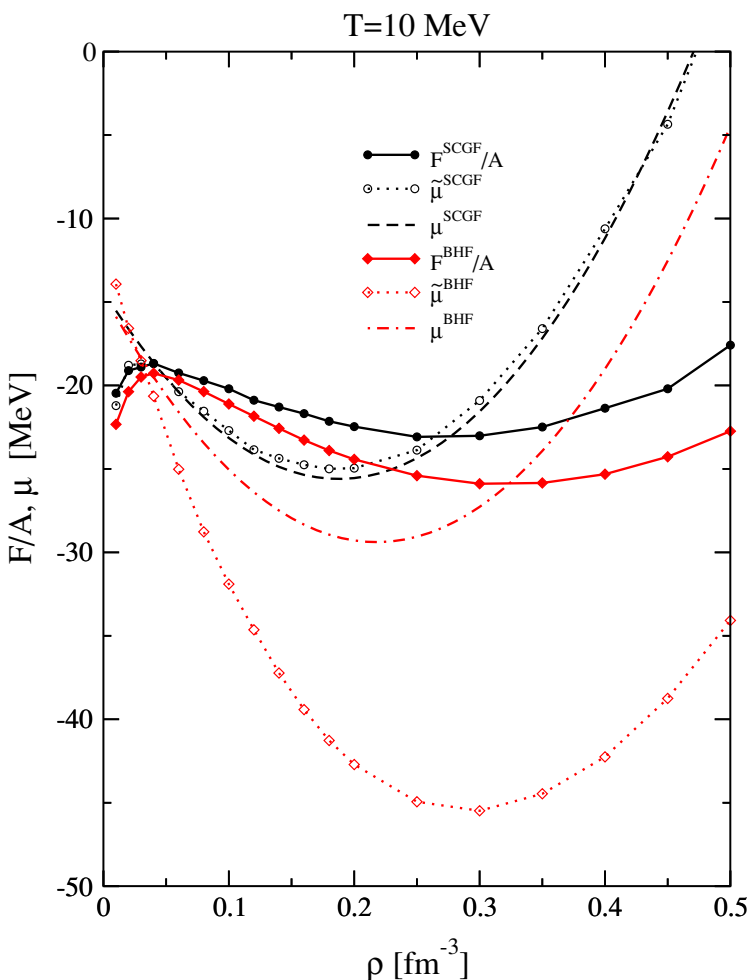

FIG. 10. (Color online) Free energies per particle (full lines) and $\tilde{\mu}$ chemical potentials (dotted lines) of the SCGF (circles) and BHF (diamonds) approaches as a function of density at a temperature of $T=10 \mathrm{MeV}$. The $\mu$ chemical potentials obtained through a numerical derivative are displayed as a dashed line for the SCGF results and as a dot-dashed line for the BHF results.

in the case of BHF, respectively. Both chemical potentials are compared with the macroscopic chemical potentials, $\mu$, obtained from the derivatives of the free energies with respect to density, Eq. (72), shown with a dashed line for the SCGF and by a dashed-dotted line for SCGF. The derivative has been performed numerically after adjusting $F$ to a third-order polynomial. Although the low-density region is not well reproduced in this rough approximation, the results of the intermediate-density region can be fully trusted and in addition they are smooth with density.

The fulfillment of TD consistency for the SCGF approach is nicely illustrated in Fig. 10. The agreement between $\tilde{\mu}$ and $\mu$ is very good above $0.05 \mathrm{fm}^{-3}$, with discrepancies of less than $1 \mathrm{MeV}$ up to $\rho=0.5 \mathrm{fm}^{-3}$. As a consequence, the Hugenholtzvan Hove theorem is also very well fulfilled, and the minimum of $F / A$ and $\tilde{\mu}$ do nicely coincide at about $\rho \sim 0.27 \mathrm{fm}^{-3}$. The situation for the BHF approach, in contrast, is much worse, as is very well known [57]. The chemical potentials $\tilde{\mu}$ and $\mu$ differ by about $10 \mathrm{MeV}$ at $\rho=0.16 \mathrm{fm}^{-3}$ and by almost $30 \mathrm{MeV}$ at the highest density considered here. In addition, the Hugenholtz-van Hove theorem is badly violated, and the value of $F / A$ at saturation differs from $\tilde{\mu}$ by about $20 \mathrm{MeV}$. Finally, we note that the propagation of holes seems to have a global repulsive effect on the free energy. Such a repulsive effect has already been detected for the energy per particle [33]. It is interesting to note, however, that the differences in free 
energies between the BHF and the SCGF are smaller than the differences in energies, because of the different entropy contributions.

The results presented here should be taken as a first step toward a full treatment of the thermal properties of infinite nuclear matter within a formalism that includes short-range correlations in a TD consistent way. From our point of view, this formalism can find several applications within the manybody and nuclear physics community. A first outcome could be, for instance, the study of the liquid-gas phase transition of symmetric nuclear matter from a realistic $N N$ potential. A critical study of the usually assumed low-temperature dependences for the relevant TD properties of matter (of the type $T^{2}$ ) could also be assessed. Other applications, such as the study of the finite-temperature equation of state, will probably demand inclusion of three-body forces in the formalism to reproduce the empirical saturation properties of nuclear matter. We expect that our results could have possible consequences for the study of intermediate-energy heavy-ion collisions [64], affecting analyses based on transport models. The use of spectral functions in the description of correlated nucleons goes beyond the quasiparticle picture customarily used in transport codes. Although some results point out that off-shell effects in the propagation of nucleons are small [65], a treatment of a kinetic equation including full spectral functions obtained from realistic $N N$ potentials (following, for instance, [66] or [67]) is, to our knowledge, still lacking. Moreover, even within the usual quasiparticle description, some of the in-medium modifications of nucleons (such as effective masses or $N N$ cross sections) are taken as simple parametrizations [68]. Our model permits the calculations of these quantities from realistic $N N$ potentials in a fully microscopical and TD consistent basis, which, properly parameterized, could be used in this kind of study.

\section{SUMMARY AND CONCLUSIONS}

The ladder or $T$-matrix approximation of the SCGF method is a $\Phi$-derivable approach and thus theoretically it should fulfill TD consistency. We have checked the numerical TD consistency of this approach for symmetric nuclear matter at finite temperature by computing an approximation to the entropy, the dynamical quasiparticle entropy $S^{\mathrm{DQ}}$, which has been discussed by Carneiro and Pethick [6]. Using this $S^{\mathrm{DQ}}$ approximation we obtain good agreement between the chemical potentials determined within the SCGF calculation and the corresponding values derived from the thermodynamical relations. Therefore, the ladder approximation of the SCGF approach supplemented by the evaluation of the entropy $S^{\mathrm{DQ}}$ provides a method of calculating thermodynamic properties of nuclear matter, which accounts for correlations beyond the mean-field picture in a consistent way.

The entropy $S^{\mathrm{DQ}}$ can be evaluated in terms of a weighting function $\mathcal{B}$, which is connected to the usual single-particle spectral function $\mathcal{A}$ of the SCGF. This means that $\mathcal{B}$ can be calculated in terms of the nucleon self-energy and an explicit evaluation of the generating functional $\Phi$ can be avoided. The $\mathcal{B}$ spectral function and its momentum, energy, density, and temperature dependences have been studied. In general, one can say that this function exhibits a more pronounced quasiparticle structure than the corresponding spectral function $\mathcal{A}$. As a consequence, the correlation effects related to the broadening of the quasiparticle peak are not very important and a quasiparticle approximation to the evaluation of the entropy is a very good approximation if the quasiparticle energies are derived from the SCGF self-energy. Even if the quasiparticle energies are approximated by the single-particle energies derived from the temperature-dependent BHF approximation, the values for the calculated entropy deviate only by as much as 10 to $20 \%$. In contrast to the SCGF, however, the BHF approximation fails to fulfill thermodynamic consistency. The microscopic and the thermodynamic chemical potential deviate substantially in the case of BHF and the Hugenholtzvan Hove theorem is violated by more than $20 \mathrm{MeV}$.

\section{ACKNOWLEDGMENTS}

The authors are very grateful to Dr. T. Frick, Dr. J. Margueron, and Dr. A. Sedrakian for useful and stimulating discussions. Arnau Rios acknowledges the support of DURSI and the European Social Funds. This work was supported by Grant Nos. FIS2005-03142 (MEC, Spain and Feder) and 2005SGR-00343 (Generalitat de Catalunya).
[1] N. F. Berk and J. R. Schrieffer, Phys. Rev. Lett. 17, 433 (1966).

[2] S. Doniach and S. Engelsberg, Phys. Rev. Lett. 17, 750 (1966).

[3] D. J. Amit, J. W. Kane, and H. Wagner, Phys. Rev. 175, 313 (1968); 175, 326 (1968).

[4] W. Brenig, H. J. Mikeska, and E. Riedel, Z. Phys. 206, 439 (1967).

[5] C. J. Pethick and G. M. Carneiro, Phys. Rev. A 7, 304 (1973).

[6] G. M. Carneiro and C. J. Pethick, Phys. Rev. B 11, 1106 (1975).

[7] D. S. Greywall, Phys. Rev. B 27, 2747 (1983).

[8] A. V. Chubukov, D. L. Maslov, and A. J. Millis, Phys. Rev. B 73, 045128 (2006).

[9] H. A. Bethe, Rev. Mod. Phys. 62, 801 (1990).

[10] Ph. Chomaz, M. Colonna, and J. Randrup, Phys. Rep. 389, 263 (2004).
[11] M. Prakash, I. Bombaci, M. Prakash, P. J. Ellis, J. M. Lattimer, and R. Knorren, Phys. Rep. 280, 1 (1997).

[12] L. P. Csernai and J. I. Kapusta, Phys. Rep. 131, 223 (1986).

[13] T. Sil, S. K. Samaddar, J. N. De, and S. Shlomo, Phys. Rev. C 69, 014602 (2004)

[14] J. Heyer, T. T. S. Kuo, J. P. Shen, and S. S. Wu, Phys. Lett. B202, 465 (1988).

[15] H. Müller and B. D. Serot, Phys. Rev. C 52, 2072 (1995).

[16] H. M. Müller, S. E. Koonin, R. Seki, and U. van Kolck, Phys. Rev. C 61, 044320 (2000).

[17] S. Fritsch, N. Kaiser, and W. Weise, Phys. Lett. B545, 73 (2002).

[18] K. A. Brueckner, C. A. Levison, and H. M. Mahmoud, Phys. Rev. 95, 217 (1954).

[19] B. D. Day, Rev. Mod. Phys. 39, 719 (1967). 
[20] C. Bloch, Nucl. Phys. 7, 451 (1958); C. Bloch and C. de Dominicis, ibid. 7, 459 (1958); 10, 181 (1959); 10, 509 (1959).

[21] M. Baldo and L. S. Ferreira, Phys. Rev. C 59, 682 (1999).

[22] A. Lejeune, P. Grange, M. Martzolff, and J. Cugnon, Nucl. Phys. A453, 189 (1986).

[23] I. Bombaci, T. T. S. Kuo, and U. Lombardo, Phys. Rep. 242, 165 (1994).

[24] B. ter Haar and R. Malfliet, Phys. Rev. Lett. 56, 1237 (1986).

[25] H. Huber, F. Weber, and M. K. Weigel, Phys. Rev. C 57, 3484 (1998).

[26] B. E. Vonderfecht, W. H. Dickhoff, A. Polls, and A. Ramos, Nucl. Phys. A555, 1 (1993).

[27] T. Alm, G. Röpke, A. Schnell, N. H. Kwong, and H. S. Köhler, Phys. Rev. C 53, 2181 (1996).

[28] P. Bożek, Nucl. Phys. A657, 187 (1999).

[29] A. Ramos, A. Polls, and W. H. Dickhoff, Nucl. Phys. A503, 1 (1989).

[30] Y. Dewulf, D. Van Neck, and M. Waroquier, Phys. Rev. C 65, 054316 (2002).

[31] P. Bożek, Phys. Rev. C 65, 054306 (2002); Eur. Phys. J. A 15, 325 (2002).

[32] O. Benhar, A. Fabrocini, and S. Fantoni, Nucl. Phys. A505, 267 (1989).

[33] T. Frick and H. Müther, Phys. Rev. C 68, 034310 (2003).

[34] T. Frick, Ph.D. thesis, University of Tübingen, 2004.

[35] T. Frick, H. Müther, A. Rios, A. Polls, and A. Ramos, Phys. Rev. C 71, 014313 (2005).

[36] V. M. Galitski and A. B. Migdal, Sov. Phys. JETP 7, 96 (1958).

[37] D. S. Koltun, Phys. Rev. C 9, 484 (1974).

[38] J. M. Luttinger and J. C. Ward, Phys. Rev. 118, 1417 (1960).

[39] V. Somà and P. Bożek, Phys. Rev. C 74, 045809 (2006).

[40] B. Vanderheyden and G. Baym, J. Stat. Phys. 93, 843 (1998).

[41] W. Weinhold, B. Friman, and W. Nörenberg, Phys. Lett. B433, 236 (1998).

[42] A. L. Fetter and J. D. Walecka, Quantum Theory of ManyParticle Systems (McGraw-Hill, New York, 1971).

[43] J. M. Luttinger, Phys. Rev. 121, 942 (1961).

[44] L. P. Kadanoff and G. Baym, Quantum Statistical Mechanics (Benjamin, New York, 1962).
[45] W. Ebeling, W. D. Kraeft, D. Kremp, and G. Röpke, Quantum Statistics of Charged Particle Systems (Akademie-Verlag, Berlin, 1986).

[46] T. Alm, B. L. Friman, G. Röpke, and H. Schulz, Nucl. Phys. A551, 45 (1993).

[47] A. Sedrakian, G. Röpke, and T. Alm, Nucl. Phys. A594, 355 (1995).

[48] A. Polls, A. Ramos, J. Ventura, S. Amari, and W. H. Dickhoff, Phys. Rev. C 49, 3050 (1994).

[49] T. Frick, H. Müther, and A. Polls, Phys. Rev. C 69, 054305 (2004).

[50] A. Rios, A. Polls, and H. Müther, Phys. Rev. C 73, 024305 (2005).

[51] G. Baym, Phys. Rev. 127, 1391 (1962).

[52] M. Cohen, Phys. Rev. 118, 27 (1960).

[53] A. Sedrakian, Prog. Part. Nucl. Phys., 58, 168 (2007).

[54] Yu. B. Ivanov, J. Knoll, and D. N. Voskresensky, Nucl. Phys. A672, 313 (2000).

[55] P. Bożek and P. Czerski, Eur. Phys. J. A 11, 271 (2001).

[56] N. M. Hugenholtz and L. Van Hove, Physica 24, 363 (1958).

[57] P. Czerski, A. DePace, and A. Molinari, Phys. Rev. C 65, 044317 (2002).

[58] R. Machleidt, F. Sammarruca, and Y. Song, Phys. Rev. C 53, R1483 (1996).

[59] H. Müther, A. Polls, and W. H. Dickhoff, Phys. Rev. C 51, 3040 (1995).

[60] Q. Chen, K. Levin, and I. Kosztin, Phys. Rev. B 63, 184519 (2001).

[61] J. Pochodzalla et al., Phys. Rev. Lett. 75, 1040 (1995).

[62] J. B. Natowitz et al., Phys. Rev. C 65, 034618 (2002).

[63] J. N. De, S. Shlomo, and S. K. Samaddar, Phys. Rev. C 57, 1398 (1998).

[64] P. Danielewicz, R. Lacey, and W. G. Lynch, Science 298, 1592 (2002).

[65] W. Cassing and S. Juchem, Nucl. Phys. A665, 377 (2000).

[66] W. Botermans and R. Malfliet, Phys. Rep. 198, 115 (1990).

[67] H. S. Köhler, Phys. Rev. C 51, 3232 (1995).

[68] P. Danielewicz, Nucl. Phys. A673, 375 (2000). 